\title{
The evolution of asteroids in the jumping-Jupiter migration model
}

\author{
Fernando Roig \\ Observatório Nacional, Rua Gal. Jose Cristino 77, Rio de Janeiro, RJ 20921-400, Brazil \\ froig@on.br \\ David Nesvorný \\ Southwest Research Institute, 1050 Walnut St., Suite 300, Boulder, CO 80302, USA \\ davidn@boulder.swri.edu
}

Received __; accepted _ 


\begin{abstract}
In this work, we investigate the evolution of a primordial belt of asteroids, represented by a large number of massless test particles, under the gravitational effect of migrating Jovian planets in the framework of the jumping-Jupiter model. We perform several simulations considering test particles distributed in the Main Belt, as well as in the Hilda and Trojan groups. The simulations start with Jupiter and Saturn locked in the mutual 3:2 mean motion resonance plus 3 Neptune-mass planets in a compact orbital configuration. Mutual planetary interactions during migration led one of the Neptunes to be ejected in less than 10 Myr of evolution, causing Jupiter to jump by about 0.3 au in semi-major axis. This introduces a large scale instability in the studied populations of small bodies. After the migration phase, the simulations are extended over $4 \mathrm{Gyr}$, and we compare the final orbital structure of the simulated test particles to the current Main Belt of asteroids with absolute magnitude $H<9.7$. The results indicate that, in order to reproduce the present Main Belt, the primordial belt should have had a distribution peaked at $\sim 10^{\circ}$ in inclination and at $\sim 0.1$ in eccentricity. We discuss the implications of this for the Grand Tack model. The results also indicate that neither primordial Hildas, nor Trojans, survive the instability, confirming the idea that such populations must have been implanted from other sources. In particular, we address the possibility of implantation of Hildas and Trojans from the Main Belt population, but find that this contribution should be minor.
\end{abstract}

Subject headings: minor planets, asteroids: general - planets and satellites: dynamical evolution and stability 


\section{Introduction}

The jumping Jupiter model is a model of planetesimal driven migration of the major planets in which an instability phase arises from the occurrence of close encounters between the planets. During this phase of close encounters, a Neptune-size planet is scattered by Jupiter, and may be ejected from the Solar System. This causes Jupiter's semi-major axis to "jump" inwards by a few tenth of au. The model was first proposed to solve some limitations of other planetary migration models, but it has not yet been fully tested against all the possible constraints imposed by many specific characteristics of the Solar System. The purpose of this paper is to investigate the effect of the jumping Jupiter evolution on the dynamics of a primordial belt of asteroids.

Planetesimal driven migration was first proposed by Fernandez \& Ip (1984) as the outcome from the gravitational scattering of a remnant disk of planetesimals by the recently formed major planets. The planets were initially assumed to be in a more compact orbital configuration. The exchange of angular momentum between the planets and the planetesimals induces a smooth change of the planets' semi-major axes resulting in a divergent migration. Smooth migration models, however, were able to explain only very few properties of the Solar System (e.g. Malhotra 1993; Liou \& Malhotra 1997). Alternatively, instability migration models have been proposed, where the planetary system evolves through a short phase of strong dynamical instability. In general, this evolution does not destabilize the major planets (e.g. Jupiter and Saturn do not suffer mutual encounters in this model), but it has deep consequences for the evolution of other Solar System populations.

The instability model was introduced by Thommes et al. (1999) and later reformulated

by Tsiganis et al. (2005), becoming known as the Nice model. In the Nice model, the instability arises from the crossing of mutual mean motion resonances between Jupiter and 
Saturn. The Nice model was able to explain the Late Heavy Bombardment (Gomes et al. 2005), and the origin of Trojan asteroids (Morbidelli et al. 2005), for example. But it failed to explain other properties, like the orbits of the terrestrial planets. This led Morbidelli et al. (2009) and Brasser et al. (2009) to propose a revision of the Nice model, in which the instability arises from the gravitational scattering of Uranus by Jupiter and Saturn. In this scenario, the successive close encounters cause sudden changes in Jupiter's (and Saturn's) semi-major axis, so the model is referred to as the "jumping Jupiter" model. A fundamental shortback of the original jumping Jupiter evolution is that, more often than not, Uranus or Neptune is ejected from the Solar Svstem. More recently, Nesvornv́ (2011) and Nesvorný \& Morbidelli (2012) attempted to solve this limitation considering systems with five initial planets: Jupiter, Saturn, and three ice giants. The fifth hypothetical planet is then ejected, leaving a system as we known today. This model has had a great success in explaining the origin of the irregular satellites of Jupiter (Nesvornv́ et al.|2014a), several properties of the Jovian satellites (Deienno et al. 2014; Nesvorný et al. 2014b), the origin of Jupiter Trojans (Nesvorný et al. 2013), and the structure of the Kuiper belt Nesvorný (2015). It has also proven to fulfill the constraints from the terrestrial planets and the architecture of the outer planets. But it has not yet been tested for the asteroid belt.

In contrast with many other populations of minor bodies, the main advantage of the asteroid belt is that it constitutes a very well known population. Its orbital distribution is little affected by observational biases, its size distribution is complete up to very small sizes, and its taxonomical distribution is well understood. This provides a number of tight constraints on the early evolution of the Solar System. In particular, we know that the asteroid belt should have suffered a significant primordial excitation, a significant mass depletion, and a significant mixture of taxonomies. Attempts to explain these issues were first due to Wetherill (1992), Petit et al. (2001), and O'Brien et al. (2007). For a detailed review, we refer the reader to Morbidelli et al. (2015). Two results are of particular relevance 
to the present work.

First, Walsh et al. (2011; 2012) proposed that the mass depletion and the primordial mixing of taxonomies in the asteroid belt could be the consequence of the early migration of Jupiter and Saturn in a gas disk (Masset \& Snellgrove 2001; Pierens et al. 2014). This evolution, which presumably happened prior to the phase of planetesimal driven migration, became known as the Grand Tack. The time elapsed between the end of the Grand Tack (i.e. end of the gas driven migration) and the occurrence of the dynamical instability is poorly constrained. It could be as short as a few Myr and as long as $\sim 500$ Myr, the latter assuming that the instability was responsible for triggering the Late Heavy Bombardment about 4 Gyr ago. Since our results have implications for the Grand Tack model, we will come back to this issue later.

Second, Minton and Malhotra (2009; 2011) addressed the stirring of the asteroids eccentricities and inclinations during the phase planetesimal driven migration. They found that in a smooth migration scenario, the characteristic time of migration should be unrealistically fast in order to let resonances sweeping to account for the orbital excitation of the asteroids. This result favors instability models alla jumping Jupiter, that could effectively provide the fast migration rates required. In particular, Morbidelli et al. (2010) applied a jumping Jupiter model with only four planets to the asteroid main belt, and found that it is possible to reproduce its current distribution in inclinations provided that the original distribution had an upper cutoff around $20^{\circ}$. We will discuss this result later in the light of our findings.

Our goal here is to analyze and discuss the significance of the jumping Jupiter migration in the evolution of the asteroid belt. We perform simulations that reproduce the current orbital distribution of the belt and allow us to constrain its initial distribution, that is, at the beginning of the planetesimal driven migration. The paper is divided as follows: 
in Sect. 2, we describe the methodology used in our simulations. In Sect. 3 we present our results, discussing the implications for the asteroid main belt, the Grand Tack model, and the Hilda and Trojan populations. Finally, in Sect. 4 we summarize our conclusions.

\section{Simulations}

We have run a series of numerical simulations of the evolution of the asteroid belt from the epoch before the triggering of the planetesimal driven migration to the present days. The asteroid belt is represented by a large number of test particles perturbed by the Jovian planets. It is worth stressing that the terrestrial planets have not been included in our simulations. The initial orbital distribution of the test particles is uniform, and the resulting final distribution is compared to the presently observed asteroid belt. This allows us to remap the initial distribution onto any desired non uniform distribution and to determine the one that provides the best fit to the current asteroid belt. We have assumed a strategy consisting in dividing the whole evolutionary process into four stages or phases, that we describe in the following.

\subsection{Phase 0: Before the instability}

This phase is intended to simulate the evolution of a primordial asteroid belt between the end of the gas driven migration (Grand Tack) and the occurrence of the jumping Jupiter instability. During this phase, the planetary system is constituted by Jupiter, Saturn and three Neptune-size ice giants. The planets do not migrate and stay in a mutual resonant

configuration that is the outcome of the previous gas driven migration phase (Pierens et al. 2014). In particular, Jupiter and Saturn are locked in the 3:2 mean motion resonance, with Jupiter slightly outside of its present orbit. The initial osculating values of semi-major axis 
$a$, eccentricity $e$, and inclination $I$ are shown in Table 1. This configuration is stable over the simulation time span of 500 Myr.

The test particles are grouped into three populations: the Main Belt, the Hilda group and the Trojan swarms. Each population is represented by 10,000 initial conditions uniformly (randomly) distributed over the following intervals:

- $1.5 \leq a \leq 5.0 \mathrm{au}$, perihelion distance $q>1.5 \mathrm{au}$, aphelion distance $Q<5.0 \mathrm{au}$, $I<30^{\circ}$, for the Main Belt (hereafter MB set);

- $4.0 \leq a \leq 4.3 \mathrm{au}, e<0.4, I<30^{\circ}$, for the Hilda group (hereafter HG set);

- $5.2 \leq a \leq 5.7 \mathrm{au}, e<0.2, I<40^{\circ}$, for the Trojan swarms (hereafter TS set).

The initial angles $M, \omega, \Omega$ (mean anomaly, argument of perihelion, and longitude of node) are randomly distributed between 0 and $360^{\circ}$ in all cases. Figure 1 shows the initial distribution of the test particles. The simulations are carried out using the SWIFT_RMVS4 symplectic integrator 1 , with a time step of $0.05 \mathrm{yr}$. The test particles are eliminated if they approach within the radius of any planet, or if $q$ gets smaller than $1 \mathrm{au}$, or if $a$ evolves beyond 100 au.

Table 2 shows the number of surviving test particles at two specific times: 10 Myr and 500 Myr. We have chosen these times because they represent the state of the asteroid belt in two interesting limits: (i) when the instability is triggered shortly after the end of the gas driven migration, and (ii) when the instability happens at a time compatible with the Late Heavy Bombardment. We note that at least half of the MB population is stable, while the HG and TS populations are significantly eroded2. This reinforces the idea that these

\footnotetext{
${ }^{1}$ https://www. boulder.swri.edu/ hal/swift.html

${ }^{2}$ It is worth noting that, due to the partial overlap of the MB and HG sets, the actual
} 
latter groups are not primordial but implanted from other sources (e.g. Levison et al. 2009; Nesvorný et al. 2013). An analysis of the reason for this erosion is beyond the scope of this paper (see Morbidelli 2002; Robutel \& Bodossian 2009).

\subsection{Phase 1: Jumping Jupiter}

The purpose of this phase is to simulate the epoch of the jumping Jupiter instability. During this phase, the five Jovian planets migrate according to three specific evolutions previously developed by Nesvorný \& Morbidelli (2012). These authors performed realistic simulations of migration, where the planets interact with a massive disk of planetesimals initially located beyond the outermost planet. In the simulations, the planets' positions and velocities were stored in a file at 1 yr intervals over a total time span of 10 Myr. We have not reproduced the simulations of Nesvorný \& Morbidelli (2012); rather we have mimicked the migration by reading the stored positions and interpolating them using an approach described in Nesvorný et al. (2013). Figure 2 shows the three evolutions we have considered, which we refer to as Cases 1, 2 and 3, respectively. The main differences between the three cases are:

- The Ice \#2 planet (middle ice giant) is ejected in Case 1 , while the Ice \#1 (innermost ice giant) is ejected in Cases 2 and 3;

- Case 2 produces a large number of encounters between Jupiter and the ejected planet, which makes Jupiter to suffer a lot of short jumps. Cases 1 and 3, on the other hand, produce less interactions and make Jupiter to experience a few longer jumps;

- In Case 1, the ejected planet reaches minimum heliocentric distances of $\sim 2$ au during

number of survivors in the Hilda region is about 1.5 times larger than in the HG set alone. 
the instability, which means that it penetrates deeply in the asteroid belt. In Case 2, the heliocentric distance of the ejected planet gets to $\sim 1.5$ au very briefly. In Case 3 , the heliocentric distances do not get much below $\sim 3$ au.

We recall that in all three cases, the planets are initially in the same orbital configuration given in Table 1. The different evolutions in each case arise from the different parameters of the planetesimal disk considered by Nesvorný \& Morbidelli (2012). The net inwards jump of Jupiter is $\sim 0.3 \mathrm{au}$ in all cases, and the instability always occurs between 5.5 and 6.5 Myr after the start of the simulations.

These three instability cases have been previously tested against a number of constraints. They satisfy the constraints defined in Nesvorný \& Morbidelli (2012), namely, the final orbits of the outer planets are similar to the real orbits. For example, the proper mode in Jupiter's orbit is excited to its present value by planetary encounters. All three cases also satisfy the terrestrial planets constraint in that Jupiter's orbit discontinuously evolves during planetary encounters (Brasser et al. 2009). This is needed to avoid secular resonances with the terrestrial planets, which would otherwise lead to a disruption of the terrestrial planets system. All three cases are equally good in explaining the capture and orbital distribution of Jupiter Troians, including their high orbital inclinations (Nesvorný et al. 2013). Case 2 shows a richer history of planetary encounters, which leads to a large perturbation of the Galilean satellite orbits that is difficult to reconcile with the present orbits of these moons (Deienno et al. 2014). Cases 1 and 3, on the other hand, have fewer planetary encounters and satisfy the Galilean satellites constraint.

The initial conditions of the test particles for Phase 1 have been cloned from the same initial conditions used for Phase 0. The reason for this is twofold: (i) we want to preserve the initial angular phases between the planets and the test particles, which is especially critical for the HG and TS populations, and (ii) we also want to increase the number 
statistics. Note, however, that we have only cloned the initial conditions of those test particles that survived Phase 0 after 10 Myr and 500 Myr, respectively. This produces two different sets of initial conditions for Phase 1. We refer to them as the "Early Instability" (EI) set and the "Late Instability" (LI) set, respectively.

For the EI set, each initial condition of Phase 0 that survived 10 Myr has been randomly cloned 15 times within an interval $\Delta a= \pm 0.001 \mathrm{au}, \Delta e= \pm 0.001$ and $\Delta I= \pm 0.1^{\circ}$ around the reference orbit. The remaining orbital elements were set equal to those of the reference orbit. After the cloning process, we have ended up with a MB population of $~ 102000$ test particles, and a HG+TS population of $\sim 15000$ test particles. For the LI set, we have applied a similar method, but only to the MB population. We have not taken into account the HG and TS populations due to the small amount of survivors at $500 \mathrm{Myr}$. We have created 21 clones of each initial condition of Phase 0 that survived after 500 Myr, ending up with a MB population of 99000 test particles. Figure 3 shows the initial distribution of the EI and LI sets. The main difference between the two sets is the larger depletion at $a>3.4$ au observed in the LI set.

The simulations have been carried out using a modified version of the SWIFT_RMVS3 symplectic integrator, that interpolates the stored planetary positions and propagates the test particles (cf. Nesvorný et al. 2013). The total time span of this phase is $10 \mathrm{Myr}$, and the integration time step is $0.05 \mathrm{yr}$. The test particles are discarded if they hit any planet, or if $q<1$ au or $a>100$ au.

\subsection{Phase 2: Residual migration}

This phase is intended to simulate the residual migration of the Jovian planets after the jumping Jupiter instability. The residual migration is a smooth process caused by the 
interaction of the planets with the disk of planetesimals. It drives the planets to reach their current orbits, making Jupiter to migrate inwards, while Saturn and the two remnant ice giants (Uranus and Neptune) migrate outwards. This migration has been simulated using a modified version of the SWIFT_RMVS4 code, that applies a non conservative acceleration to each planet. The non conservative acceleration $\boldsymbol{a}$ has the form:

$$
\boldsymbol{a}=\alpha \exp \left(-\frac{t}{\tau}\right) \boldsymbol{v}-2 \eta \frac{\boldsymbol{r} \cdot \boldsymbol{v}}{r^{2}} \boldsymbol{r}
$$

where $t$ is the time, $\boldsymbol{r}, \boldsymbol{v}$ are the position and velocity vector of the planet, and $\alpha, \tau, \eta$ are constants specific to each planet. The first term provides a smooth drift in semi-major axis, while the second term produces damping in eccentricity. An additional term of the form $-2 \zeta v_{z}$ has been introduced in the $z$ component of the acceleration to produce inclination damping. The values of $\alpha, \tau, \eta, \zeta$ were tuned to get each planet in its present orbit at the end of the simulation. The initial conditions for both the planets and the test particles have been taken directly from the final conditions produced by Phase 1. An example of the initial conditions is shown in Fig. 4. The most notable feature in this figure is the gap around 2 au that has been opened by the $\nu_{6}$ secular resonance; this resonance is located more or less at its present location (Knežević et al. 1991) already at the end of Phase 1.

As in previous phases, the test particles are discarded if they hit any planet, or if $q<1$ au or $a>100 \mathrm{au}$. The total time span of this phase is $100 \mathrm{Myr}$, and the integration time step is $0.05 \mathrm{yr}$.

\subsection{Phase 3: Long term evolution}

This is the last stage of our simulations. It intends to reproduce the evolution of the asteroid belt from the end of the planetesimal driven migration to the present days. The planets do not migrate any more, and their initial conditions are those resulting from Phase 
2. The total time span of this phase is 4 Gyr. The evolution has been simulated using the SWIFT_RMVS4 code, with a time step of $0.05 \mathrm{yr}$.

The initial conditions for the test particles have been taken from the final conditions produced by Phase 2. However, at the end of Phase 2 there are typically between 25000 and 50000 surviving test particles, depending on the migration case and on the EI or LI set. Since it is not possible to simulate such amount of test particles over 4 Gyr in a reasonable CPU time, we have performed a down-sampling of the sets. The down-sampling process involves the following steps:

- test particles with $q<1.6$ au and $a<2.1$ au are not considered at all. This is because we are not interested at this time in the Mars crossing population $(q<1.6 \mathrm{au})$, nor in the particles that survive in the Extended Inner Belt $(a<2.1 \mathrm{au}$; Bottke et al. 2012). This procedure typically removes $\sim 30 \%$ of the test particles from the whole sample. In principle, these test particles should have been significantly depleted during previous phases if the terrestrial planets were included in the simulations. Actually, the only remnant of this population that we recognize today is the Hungaria group, whose analysis is beyond the scope of the present work 3 .

- test particles with $3.5 \leq a \leq 5.3$ au are all considered. This is because this region is significantly depleted during the previous phases and we want to keep track of the few survivors, especially at the HG and TS population (see Sect. 3.5). Typically, these test particles represent less than $0.3 \%$ of the whole sample.

- the remaining test particles with $a<3.5$ au are selected at random, producing a down-sampled set with only 1008 initial orbits. This implies that the set is

\footnotetext{
${ }^{3}$ The Hungaria group is above the absolute magnitude limit imposed to our comparison population in Sect. 3 .
} 
down-sampled by a factor $f_{\text {down }}$ between 15 and 35, depending on the migration case and on the EI or LI set.

Note that this approach assigns a larger weight to test particles with $a \geq 3.5$ au than to the rest of the simulated particles. Nevertheless, this does not introduce any appreciable bias in our results, because these particles contribute very little to the distribution of the down-sampled set (see Sect. 33).

An example of the down-sampling procedure is shown in Fig. 5, Looking at the cyan dots in this figure, we can see that the main features of the present main belt have been already sculpted at the end of Phase 2. In particular, we can clearly see the gaps opened by the $\nu_{6}$ and $\nu_{16}$ secular resonances (indicated by dashed lines), as well as the Kirkwood gaps opened at the 3:1, 5:2. 7:3 and 2:1 mean motion resonances with Jupiter (indicated at the top of the plot).

During Phase 3, each initial set of 1008 test particles has been propagated together with the four Jovian planets. Depending on the migration case and the EI or LI set, between $15 \%$ and $25 \%$ of the particles become discarded by the same reasons as in the previous phases.

\section{Results}

We have compared the final state of the test particles at the end of Phase 3 with the current distribution of the real asteroid belt. We have taken into account the 574 asteroids with $q \geq 1.6 \mathrm{au}, a \leq 4.2 \mathrm{au}$, and absolute magnitude $H \leq 9.7$, that corresponds to diameters larger than 40-70 km depending on the assumed albedo. The magnitude limit

is the same considered by Morbidelli et al. (2010). The upper cutoff in $a$ has been adopted to exclude the Trojan population from the comparison. These asteroids may be considered 
"primordial" in the sense that they have not suffered catastrophic collisions over the age of the Solar System (Farinella \& Davis 1992). They have not suffered significant variations of their orbital elements either, especially in terms of semi-major axis, because they are too big to be affected by the Yarkovsky effect (Bottke et al. 2006). Moreover, these asteroids constitute an observationally complete sample.

Figure 6 shows the distribution of the 574 real asteroids together with the distribution of the 800 test particles with $q \geq 1.6$ au and $a \leq 4.2$ au from the EI set, that survived at the end of Phase 3 in migration Case 14 . We can see a quite good general agreement between the two distributions. A more detailed way to compare the results is to look at the cumulative distributions in $e$ and $I$, and the density distribution in $a$. This is shown in Fig. 7. As stated before, we have verified that the region between 3.5 and 4.2 au does not contribute significantly to the overall statistics. In fact, this region accounts for only $3 \%$ of the real population considered here, and between $0.5 \%$ and $3 \%$ of the test particles (depending on the migration case and the EI or LI sets).

In Fig. 7, we note that there is a clear excess of high eccentricity $(e>0.25)$ and high inclination $\left(I>20^{\circ}\right)$ test particles with respect to the real asteroid belt. In particular, we have verified that the high inclination particles had high inclinations already at the beginning of Phase 0. Therefore, this excess could be eliminated, for example, by applying a suitable upper cutoff to the initial population. More realistic initial distributions can be tested to find the one that best matches the real asteroid belt, as we show in the following.

\footnotetext{
${ }^{4}$ It is worth noting that, at the end of Phase 3, Jupiter is at $a \sim 5.1 \mathrm{au}$, that is a little bit inwards than the actual location $(a \simeq 5.2 \mathrm{au}$ ). This produces a shift of all the resonance locations (Kirkwood gaps) in our simulated test particles. To correct this, we have applied an appropriate shift in the semi-major axis of the particles to get the Kirkwood gaps in their right position.
} 
Our first goal has been to match the cumulative distributions in $e$ and $I$; the differential distribution in $a$ is considered later (Sect. 3.4). The procedure involved to remap the uniform initial orbital distribution of the test particles into a non uniform distribution by selecting appropriate initial orbits. Then, the final state of these selected orbits was used to match the observations. For this purpose, we have tested four possible types of non uniform initial density distributions:

- upper cutoff distribution

$$
p(x)= \begin{cases}1 & \text { if } x \leq c \\ 0 & \text { if } x>c\end{cases}
$$

parametrized by the cutoff $c$ (with $x \equiv e, I$ );

- Rayleigh distribution

$$
p(x)=\frac{x}{\gamma^{2}} \exp \left(-\frac{x^{2}}{2 \gamma^{2}}\right)
$$

parametrized by the mode $\gamma$;

- Maxwell distribution

$$
p(x)=\sqrt{\frac{2}{\pi}} \frac{x^{2}}{\beta^{2}} \exp \left(-\frac{x^{2}}{2 \beta^{2}}\right)
$$

parametrized by the mode $\sqrt{2} \beta$; and

- Gaussian distribution

$$
p(x)=\frac{1}{\sigma \sqrt{2 \pi}} \exp \left(-\frac{(x-\mu)^{2}}{2 \sigma^{2}}\right)
$$

parametrized by the mean $\mu$ and the standard deviation $\sigma$.

Assuming a given type of initial distribution for $e$, and a given type of initial distribution for $I$ (not necessarily the same), we have scanned all the possible values of the distribution parameters. Then, we have applied a Kolmogorov-Smirnoff (K-S) test to compare the final cumulative distributions in $e$ and $I$ obtained for each value of the parameters, with the real 
asteroid belt. The results are presented in the form of color maps, where the color level indicates the value of the K-S statistic $D_{\mathrm{KS}}$. In these maps, the horizontal axis gives the value of the parameter for the assumed initial distribution in $e$. The vertical axis gives the value of the parameter for the assumed initial distribution in $I$. The white curve encloses the region where $D_{\mathrm{KS}}$ has the highest significance level (Press et al. 1992). We recall that in the case of upper cutoff, Rayleigh and Maxwell distributions, there is only one parameter to vary. In the case of the Gaussian distribution, we have fixed one of the parameters (either $\mu$ or $\sigma$ ) and let the other vary.

\subsection{The Early Instability set}

Figure 8 shows the results from the K-S test applied to the two dimensional distributions in the $e, I$ plane, like the one shown in Fig. 6r. We show examples using the initial upper cutoff and Rayleigh distributions. Best fits with $D_{\mathrm{KS}} \leq 0.1$ have been found for migration Cases 1 and 3. For migration Case 2, it has not been possible to get a good fit because there is always an excess of high eccentricity test particles. This indicates that test particles in Case 2 always get too much excited in $e$, but not necessarily in $I$. It is interesting to recall that Case 2 have also had a bad performance in the simulations of the Galilean satellites by Deienno et al. (2014), because the orbits of these satellites get too much excited. We can see that, considering upper cutoff distributions, the primordial main belt should have been quite exited in $I$ (up to $20^{\circ}$ ) and $e$ (up to 0.2). The upper limit in inclinations is compatible with the findings by Morbidelli et al. (2010). Considering Rayleigh distributions, the $e$ and $I$ should have been peaked at $\sim 0.1$ and $\sim 10^{\circ}$, respectively. A similar result is obtained considering Maxwell distributions. In any case, the best fit values are consistent with the mean values of $e, I$ in the current main belt. This result supports the idea that the presently excited values of eccentricities and inclinations should have mostly been acquired 
before the phase of planetesimal driven migration, for example, in the Grand Tack model. The jumping Jupiter instability and the subsequent residual migration of the outer planets help to disperse even more the already excited $e$ and $I$, and to sculpt the main belt into its current shape (e.g. Fig. 5).

\subsection{The Late Instability set}

Figure 9 is similar to Fig. 8, but for the LI set. Only results for Cases 1 and 3 are presented, since once again Case 2 has been unable to provide a reasonable good fit. We have not found any significant differences with respect to the EI set, except for a slightly tighter constraint of the best fits. This figure also shows that, in general, Maxwell distributions generate tighter constraints than Rayleigh (also observed in the EI set). We can conclude that, concerning the distributions in $e$ and $I$, there is no evidence that favors the model of Early instability over the Late one, or vice-verse. This is expected since the main difference between the EI and LI sets concerns the stronger depletion of the outer belt (Fig. 3), beyond the location of the 2:1 mean motion resonance, that has a small weight in the whole $e, I$ distributions.

\subsection{Implications for the Grand Tack}

The Grand Tack is a specific case of gas driven migration of the Jovian planets. It was originally proposed to explain the low mass of Mars and the current location of Jupiter beyond the ice line (Walsh et al. 2011). In this model, Jupiter and Saturn formed beyond the ice line and started to migrate inwards due to the torque exerted by the gas disk (type II migration). Saturn migrated faster than Jupiter, and when Jupiter had reached $\sim 1.5$ $\mathrm{au}$, both planets became captured and locked in a mutual 3:2 mean motion resonance. At 
this point, the interaction of the resonant configuration with the gas torque reverted the migration, and both planets started to drift outwards (Masset \& Snellgrove 2001). By the time Jupiter reached $\sim 5.5 \mathrm{au}$, the gas had already dissipated and the type II migration stopped.

During the Grand Tack, Jupiter crosses the asteroid belt first inwards and then outwards (Walsh et al. 2012). Therefore, this model has deep consequences for the evolution of asteroids. In particular, the Grand Tack is thought to be responsible for: (i) the significant mass depletion of the asteroid belt, (ii) the primordial excitation of the asteroids' eccentricities and inclinations, and (iii) the partial mixing of taxonomic classes (especially the S-type and C-type) in the main belt (for a detailed review see Morbidelli et al. 2015).

According to Walsh et al. (2012), the Grand Tack would produce an asteroidal population that can be reasonably well approximated by a Gaussian distribution in $e$ and a Rayleigh distribution in $I$. The best fit Gaussian distribution has mean $\mu \simeq 0.38$ and standard deviation $\sigma \simeq 0.17$, while the best fit Rayleigh distribution has mode $\gamma \simeq 10^{\circ}$. Using these values, we may therefore test whether the initial distributions predicted by the jumping Jupiter instability are compatible with the expectation for the distributions from the Grand Tack.

Figure 10 shows the result. This figure is similar to Figs. 8 and 9 , except that the axes correspond to initial distributions like those of the Grand Tack. This figure corresponds to the EI set; a quite similar result has been obtained for the LI set. Once again, migration Case 2 (not shown here) has been unable to provide a good fit due to an excess of high eccentricity test particles in the final distribution.

We note that, concerning the inclinations, good fits to the current asteroid belt are obtained for a Rayleigh $\gamma \sim 10^{\circ}$ that is compatible with the Grand Tack nominal value. For the eccentricities, good fits are obtained with $\mu \sim 0.1$ and $\sigma \sim 0.1-0.3$. However, if we 
force $\mu$ to have a large value, we need also a large value of $\sigma>0.35$. On the other hand, if we force $\sigma$ to have a moderate value, we need a small value of $\mu<0.15$. This result is not compatible with the nominal values derived Walsh et al. (2012). Actually, our simulations using the nominal values produce a final distribution with a clear excess of high $e$ particles, as shown in Fig. 11.

There are some possible explanations for this problem with eccentricity:

- the distribution produced by the Grand Tack is correct, but the population becomes significantly depleted at the high eccentricities $(e>0.2)$ by some mechanism not accounted for in our simulations. This depletion might be caused, for example, by the terrestrial planets;

- the distribution produced by the Grand Tack is not correct, and either the model overestimates the stirring of eccentricities (i.e. $\mu$ is too large), or underestimates its spreading (i.e. $\sigma$ is too small);

- the Jovian planets evolved in a different way than considered in our study, thus leading to different results than presented here.

An analysis of these possibilities is beyond the scope of this paper, but the possible effect of the terrestrial planets is worth of discussion.

On one hand, the terrestrial planets might be responsible for a pre-instability depletion of the high eccentricity asteroids, that would result in an initial distribution peaked at low $e$ and with a moderate spreading, as required (see Fig. 10, middle column). This would put a strong constraint on the timing of the instability, because: (i) the terrestrial planets were almost certainly not completely formed at $10 \mathrm{Myr} 5$ after the beginning of Phase 0 ,

\footnotetext{
${ }^{5}$ For example, the Moon-forming impact was dated to have happened at $\sim 30-50 \mathrm{Myr}$.
} 
and (ii) even if they would be present at the beginning of Phase 0 , it is quite unlikely that they produce a significant depletion of the belt in only 10 Myr of evolution. In any case, a pre-instability depletion model seems to favor a situation where the instability happens at later times. Actually, simulations by Deienno et al. (2015, in preparation) indicate that an initially cool system of terrestrial planets would require $\sim 200 \mathrm{Myr}$ to deplete the main belt so as to shift the eccentricity peak of the Grand Tack distribution from 0.38 to $\sim 0.2$ before the instability. These authors also found that the current eccentricities and inclinations of the asteroid belt are quite compatible with the Grand Tack initial distribution, except at the very low values of $e$ and $I$. Their model accounted for the terrestrial planets, but they considered a simplified model of the jumping Jupiter instability, in which the orbits of the major planets are artificially moved (instantaneously) from their pre-instability configuration to their present configuration.

On the other hand, the terrestrial planets may be responsible for a post-instability depletion, since they will certainly continue to erode the main belt at the high eccentricities after the instability and until the present times. In this model, the instability may happens at earlier times, and the main belt may still loose the necessary fraction of high eccentricity orbits so as to reach the present distribution.

In the end, although including the terrestrial planets might help to conciliate our results with the Grand Tack model, they would not allow us to decide between an early vs. late instability model.

\subsection{The distribution in semi-major axis}

Once we have determined the best fit parameters for the distribution in $e$ and $I$, we may now pay attention to the distribution in $a$. Analyzing the histograms like the one in 
Fig. 7, we conclude that our simulations have been able to reasonably reproduce the overall distribution in $a$ of the current asteroid belt. Migration Case 1 gives slightly better results than Cases 2 and 3. We have not found significant differences between the EI and LI sets. We also realized that the distribution in $a$ is nearly independent on the assumed initial distributions in $e$ and $I$.

A major discrepancy between our simulations and the real asteroid belt occurs in the interval $2.8<a<3.0$ au. Today, this region of the belt shows a much lower density of asteroids compared to the neighboring regions. The reason for this is still unclear (e.g. Minton \& Malhotra 2009). Brož et al. (2013) refer to this region as the "pristine zone". In our simulations, we have not been able to reproduce the low density of the pristine zone. Actually, in some simulations the density we have obtained can be up to twice the observed one.

Here, we test whether a primordial depletion of this zone (i.e. before the jumping Jupiter instability) could explain the current density. We do not intend to provide a dynamical explanation for such hypothetical depletion, but simply to determine if it could be a plausible alternative. The depletion has been simulated by remapping the initial density distribution in $a$ into a "square band cut" distribution, i.e.:

$$
p(a)= \begin{cases}1 & \text { if } a<a_{\min } \text { or } a>a_{\max } \\ \varepsilon & \text { if } a_{\min } \leq a \leq a_{\max }\end{cases}
$$

with $0 \leq \varepsilon \leq 1$ ( $\varepsilon=0$ means that all the test particles within the band are removed). The final density distribution obtained from the remapped initial distribution has been fitted to the real asteroid belt applying a $\chi^{2}$ test. We have considered a band cut distribution with a fixed band width $a_{\max }-a_{\min }=0.15 \mathrm{au}$, compatible with the current width of the pristine zone. The band center $a_{\mathrm{c}}$ and the cut level $\varepsilon$ have been taken as free parameters of the fit. Figure 12 shows the results for the EI set in the three migration cases. The 
color scale gives the values of $\chi^{2}$, and we have considered that good fits are obtained for $\chi^{2} \leq 0.14$ (dark blue). In these examples, we have assumed that $e$ and $I$ initially followed the Rayleigh distributions with $\gamma=0.1$ and $10^{\circ}$, respectively, corresponding to the best fits for these orbital elements.

In Case 1 , we observe that $\varepsilon \lesssim 0.3$ (i.e. $\gtrsim 30 \%$ of depletion) around 2.85 au (black rectangle) appears to provide slightly better fits to the $a$ distribution. However, the results are inconclusive and, in principle, good fits could also be obtained without any depletion at all (i.e. $\varepsilon=1$ ). An example is shown in Fig. 13, This is the same simulation shown in Fig. 7, but remapping the initial distributions in $e$ and $I$ only. Apart from the very good matches in the $e$ and $I$ distributions, as expected, we see that the distribution in $a$ shows a better match in the pristine zone, even without having applied any remapping to its initial distribution. On the other hand, in Case 2 no good fit can be found in spite of the application of a band cut distribution in $a$. Finally, in Case 3, an initial band cut distribution in $a$ produces a much worse fitting than the original distribution. The reason for these differences among the three cases may be related to the amount of dispersion in $a$ caused in each migration model. Table 3 shows the median, mean and maximum dispersion in orbital elements obtained in the different cases. These values are reflecting the behavior of the fifth planet in each simulation, as discussed in Sect. 2.2 (Brasil et al., 2015, in preparation). It appears that Case 2 causes too much dispersion in $a$ as to blur any structure in the initial distribution. On the other hand, Case 3 does not disperse enough the semi-major axes, and any sharp structure in the initial distribution will remain in the final distribution. For example, applying a large initial depletion around 2.6 au leaves this region almost depleted over the whole simulation (because it is not replenished by particles dispersed from the neighboring regions), thus producing the large $\chi^{2}>0.25$ values observed in Fig. 12, 
The values of $\delta a$ shown in Table 3 also allow us to infer that the dispersion caused by the jumping Jupiter instability would not be enough to produce a significant mixing of the taxonomic classes in the main belt, as suggested by the observational evidence (Gradie

\& Tedesco 1982; DeMeo \& Carry 2013, 2014). This supports the idea that the mixing of taxonomies is the consequence of a pre-instability mechanism, as for example, the Grand Tack model.

\subsection{The Hilda and Trojan populations}

As discussed in Sect. 2.1, any primordial population of Hildas and Trojans that might have existed after the Grand Tack, is strongly depleted already during Phase 0. Even in the most optimistic model of an Early Instability, the HG is reduced to $\sim 10 \%$, and the TS to less than 1\% (Table 2). We have also verified that these remnant populations do not survive the jumping Jupiter instability. Of the initially 15000 test particles in the HG and TS populations at the beginning of Phase 1 (red dots in Fig. 3), less than 1\% have survived the instability but all of them have been scattered out of the 3:2 and 1:1 mean motion resonances with Jupiter. We conclude that in an Early Instability hypothesis, the survival probability of a post Grand Tack population is $<10^{-5}$ for the HG and $<10^{-6}$ for the TS. These probabilities reduce further by two orders of magnitude in a Late Instability hypothesis.

On the other hand, we have realized that after the instability a number of MB test particles have became apparently implanted in the region of the HG and TS populations. An example is shown in Fig. 14, top row, where the blue dots correspond to the test particles that survived in the HG and TS regions at the end of Phase 1. We have also verified that most of these implanted test particles had initial orbits at the beginning of Phase 1 with $a>3$ au (Fig. 14, bottom row). Since Jupiter is at $\sim 5.5$ au before the 
instability, this source region is beyond the 5:2 mean motion resonance with Jupiter, i.e. it is equivalent to the current outer main belt. Finally, our simulations also indicate that less than $7 \%$ of the implanted populations have survived the phase of residual migration and are still active at the end of Phase 3 (red dots in Fig. 14). This allows us to make an estimation of the implanting probability, simply dividing the number of surviving test particles at the end of Phase 3 by the total number of test particles with $a>3$ au at the beginning of Phase 1 (it is worth recalling that the implanted test particles have not been down-sampled in the transition from Phase 2 to Phase 3). The estimated probability for implanting $\mathrm{HG}$ particles is $\sim 4 \times 10^{-4}$ in the most optimistic case (EI set, migration Case 3 ) and $<4 \times 10^{-5}$ in the most pessimistic case (LI set, migration Case 1). For implanting TS particles, the probability is $<10^{-5}-10^{-6}$.

In order to estimate the current fraction of Hilda asteroids that could have been implanted from the MB, we have proceeded as follows:

1. we have verified that, in general, our simulations reproduce quite well the distribution of asteroids in the interval $3.2 \leq a \leq 3.5$ au (see for example Figs. 7k, 11k and 13c), at least in terms of relative density. This region is presently occupied by the group of Cybele, and we will refer to it as the Cybele region;

2. we have also verified that the test particles that have survived in the Cybele region have originated in the same region as the implanted Hildas, i.e. our simulated Cybeles had $a>3$ au at the beginning of Phase 1 ;

3. if $n_{C}$ is the number of Cybeles and $n_{H}$ is the number of Hildas that have survived in our simulations at the end of Phase 3 , then the present fraction of possibly implanted Hildas can be computed as:

$$
f_{\text {imp }}=\frac{n_{H}}{f_{\text {down }} n_{C}} \frac{N_{C}}{N_{H}}
$$


where $f_{\text {down }}$ is the down sampling factor that has been applied to the Cybele region, $N_{C}$ is the current number of asteroids in the Cybele region, and $N_{H}$ is the current number of Hilda asteroids (both with $H<11$, corresponding to diameter greater than $\sim 40 \mathrm{~km}$ for albedo $\sim 0.05)$.

We computed that $f_{\text {imp }}<5 \%$ in the simulations with the EI set, and $f_{\text {imp }}<1 \%$ in the simulations with the LI set. For the Trojans, these fractions are two orders of magnitude smaller. This result has implications for the current distribution of taxonomical classes among the Hildas. Assuming that the source of the implanted Hildas is dominated by C-type asteroids, as is the case of the current outer main belt, we could expect to find today 1 or 2 implanted C-type Hildas with $H<11$. Actually, up to this magnitude limit, the Hilda group is dominated by P-type and D-type asteroids (Dahlgren \& Lagerkvist 1995; Dahlgren et al. 1997)6, and only one C-type asteroid is known: (334) Chicago.

The above results support the idea that current Hildas and Trojans are not primordial, but were captured from other Solar System populations during the migration instability. Morbidelli et al. (2005) and Nesvorný et al. (2013) have shown that Trojans can be captured from the disk of planetesimals initially located beyond the orbit of Neptune. It is possible that Hilda asteroids could also be captured from this population (Levison et al. 2009), but, at variance with Trojans, a small fraction $(<5 \%)$ of them could have also been captured from the outer asteroid main belt.

\section{Conclusions}

In this work, we have investigated the evolution of the asteroid belt during the jumping Jupiter instability related to the planetesimal driven migration of the Jovian planets. We

${ }^{6}$ See also http://ssd.jpl.nasa.gov/sbdb_query.cgi 
have simulated the dynamical behavior of test particles from the epoch before the instability to the present days. We have considered sets of initial conditions distributed uniformly, that have been remapped to different non uniform distributions in order to find a best match between the final results and the current asteroid belt. Our conclusions can be summarized as follows:

- we have not found any indication that favors a model in which the instability occurs early (soon after the end of the gas driven migration), with respect to a model in which the instability occurs later (at a time compatible with the Late Heavy Bombardment epoch);

- of the three models of jumping Jupiter instability that we have tested, the one identified as Case 2 has been unable to produce any good fit to the present asteroid belt, because the final eccentricities get too much excited;

- in the other two models (Cases 1 and 3), the best fits indicate that, prior to the instability, the asteroid belt should have been quite excited in eccentricities (0.1 to 0.2 ) and inclinations $\left(10^{\circ}\right.$ to $\left.20^{\circ}\right)$. The main effect of the jumping Jupiter instability is only to cause a moderate dispersion of the $e, I$ values. This supports the idea that most of the current excitation of the main belt occurred before the instability, as it would be the case of the Grand Tack model;

- concerning the upper limit for the initial inclination distribution, we have found the same results as Morbidelli et al. (2010);

- our results predict that before the instability, the asteroid belt should have had a Rayleigh (or Maxwell) distribution in inclinations peaked at $\sim 10^{\circ}$. This is compatible with the value found by Walsh et al. (2012) for the distribution resulting from the Grand Tack model; 
- on the other hand, our results predict that the eccentricities of the pre-instability asteroid belt should have been peaked at $\sim 0.1$, which is smaller than the value of 0.38 assumed by Walsh et al. (2012). This discrepancy could be resolved invoking an additional mechanism that would deplete the high $e$ orbits during the transition between the Grand Tack and the jumping Jupiter instability. In principle, the terrestrial planets, which have not been considered in our simulations, could account for such depletion;

- our model is able to reproduce quite well the density distribution in semi-major axis of the main belt up to $3.5 \mathrm{au}$, except in the the interval between 2.8 and $3.1 \mathrm{au}$, where we have only been able to partially reproduce the very small density observed today. This could be indicating that such region became even more depleted by some other (not yet understood) mechanism after the jumping Jupiter instability;

- the median and mean dispersion in semi-major axis caused by the instability does not seem to be enough to provide the required mixing of taxonomic classes in the main belt;

- the jumping Jupiter instability is able to implant asteroids from the outer main belt into the Hilda group with a very low probability, although enough to explain the presence of a few big Hildas belonging to the $\mathrm{C}$ taxonomic class.

We wish to thank the anonymous referee for his helpful comments and suggestions. This work has been supported by the Brazilian National Research Council (CNPq) through fellowship 312292/2013-9 and grant 401905/2013-6 within the Science Without Borders Program, and by NASA's Solar System Workings program. Simulations has made use of: (i) the computing facilities of the Laboratory of Astroinformatics (IAG/USP, NAT/Unicsul), supported by FAPESP grant 2009/54006-4 and INCT-A, (ii) the Pleiades Supercomputer 
of NASA's High-End Computing Capability, and (iii) the cluster of the Department of Astronomy of the National Observatory of Rio de Janeiro, acquired through CAPES grant $23038.007093 / 2012-13$ 


\section{REFERENCES}

Bottke, W. F., Vokrouhlický, D., Minton, D., et al. 2012, Nature, 485, 78

Bottke, Jr., W. F., Vokrouhlický, D., Rubincam, D. P., \& Nesvorný, D. 2006, Ann. Rev. Earth Planet. Sci., 34, 157

Brasser, R., Morbidelli, A., Gomes, R., Tsiganis, K., \& Levison, H. F. 2009, A\&A, 507, 1053

Brož, M., Morbidelli, A., Bottke, W. F., et al. 2013, A\&A, 551, A117

Dahlgren, M., \& Lagerkvist, C.-I. 1995, A\&A, 302, 907

Dahlgren, M., Lagerkvist, C.-I., Fitzsimmons, A., Williams, I. P., \& Gordon, M. 1997, A\&A, 323, 606

Deienno, R., Nesvorný, D., Vokrouhlický, D., \& Yokoyama, T. 2014, AJ, 148, 25

DeMeo, F. E., \& Carry, B. 2013, Icarus, 226, 723

-. 2014, Nature, 505, 629

Farinella, P., \& Davis, D. R. 1992, Icarus, 97, 111

Fernandez, J. A., \& Ip, W.-H. 1984, Icarus, 58, 109

Gomes, R., Levison, H. F., Tsiganis, K., \& Morbidelli, A. 2005, Nature, 435, 466

Gradie, J., \& Tedesco, E. 1982, Science, 216, 1405

Knežević, Z., Milani, A., Farinella, P., Froeschlé, C., \& Froeschlé, C. 1991, Icarus, 93, 316

Levison, H. F., Bottke, W. F., Gounelle, M., et al. 2009, Nature, 460, 364

Liou, J. C., \& Malhotra, R. 1997, Science, 275, 375 
Malhotra, R. 1993, Nature, 365, 819

Masset, F., \& Snellgrove, M. 2001, MNRAS, 320, L55

Minton, D. A., \& Malhotra, R. 2009, Nature, 457, 1109

-. 2011, ApJ, 732, 53

Morbidelli, A. 2002, Modern celestial mechanics: aspects of solar system dynamics (London: Taylor \& Francis), ISBN 0415279399

Morbidelli, A., Brasser, R., Gomes, R., Levison, H. F., \& Tsiganis, K. 2010, AJ, 140, 1391

Morbidelli, A., Brasser, R., Tsiganis, K., Gomes, R., \& Levison, H. F. 2009, A\&A, 507, 1041

Morbidelli, A., Levison, H. F., Tsiganis, K., \& Gomes, R. 2005, Nature, 435, 462

Morbidelli, A., Walsh, K. J., O’Brien, D. P., Minton, D. A., \& Bottke, W. F. 2015, ArXiv e-prints, arXiv:1501.06204

Nesvorný, D. 2011, ApJ, 742, L22

—. 2015, ArXiv e-prints, arXiv:1506.06019

Nesvorný, D., \& Morbidelli, A. 2012, AJ, 144, 117

Nesvorný, D., Vokrouhlický, D., \& Deienno, R. 2014a, ApJ, 784, 22

Nesvorný, D., Vokrouhlický, D., Deienno, R., \& Walsh, K. J. 2014b, AJ, 148, 52

Nesvorný, D., Vokrouhlický, D., \& Morbidelli, A. 2013, ApJ, 768, 45

O’Brien, D. P., Morbidelli, A., \& Bottke, W. F. 2007, Icarus, 191, 434

Petit, J.-M., Morbidelli, A., \& Chambers, J. 2001, Icarus, 153, 338 
Pierens, A., Raymond, S. N., Nesvorný, D., \& Morbidelli, A. 2014, ApJ, 795, L11

Press, W. H., Teukolsky, S. A., Vetterling, W. T., \& Flannery, B. P. 1992, Numerical Recipes: The Art of Scientific Computing, Second Edition, Vol. 1 (Cambridge University Press)

Robutel, P., \& Bodossian, J. 2009, MNRAS, 399, 69

Thommes, E. W., Duncan, M. J., \& Levison, H. F. 1999, Nature, 402, 635

Tsiganis, K., Gomes, R., Morbidelli, A., \& Levison, H. F. 2005, Nature, 435, 459

Walsh, K. J., Morbidelli, A., Raymond, S. N., O'Brien, D. P., \& Mandell, A. M. 2011, Nature, 475, 206

—. 2012, Meteor. Planet. Sci., 47, 1941

Wetherill, G. W. 1992, Icarus, 100, 307 
Fig. 1.- Distribution of the test particles at the beginning of Phase 0. Black dots represent the main belt (MB) population. Red dots represent the Hilda group $(\mathrm{HG})$ centered at $\sim 4.1$ $\mathrm{au}$, and the Trojan swarms (TS) centered at $\sim 5.4$ au. Note that the positions of these populations are moved outwards with respect to their present locations, since the 3:2 and 1:1 mean motion resonances with Jupiter are shifted to larger semi-major axes. The big black dot represents the initial position of Jupiter.
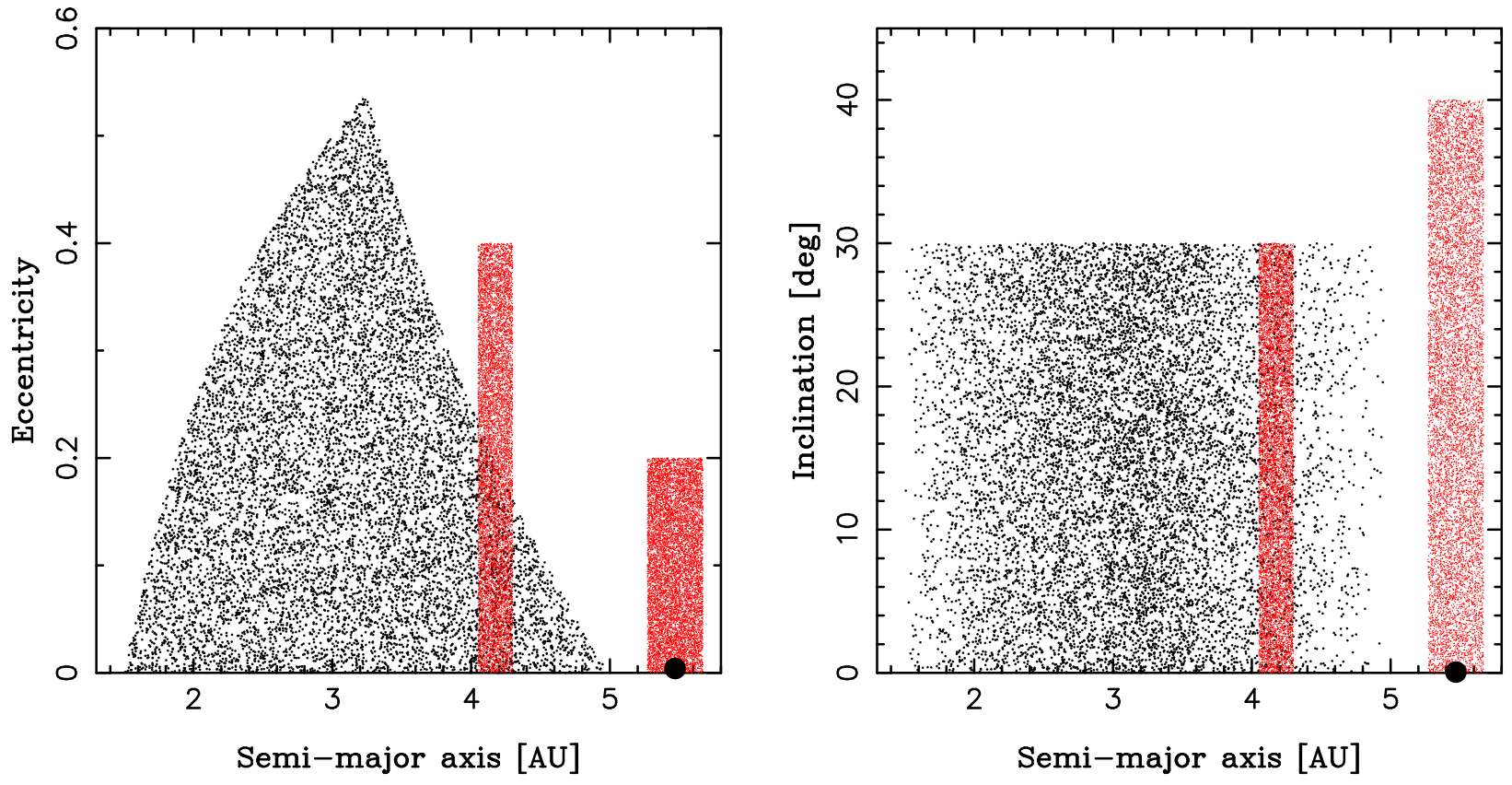
Fig. 2.- The three cases of jumping Jupiter instability from Nesvorný et al. (2013) that are studied in this paper. For each planet, the plots show the semi-major axis and the perihelion and aphelion distances. Black corresponds to Jupiter, red to Saturn, and green, blue and cyan to the three icy giants.
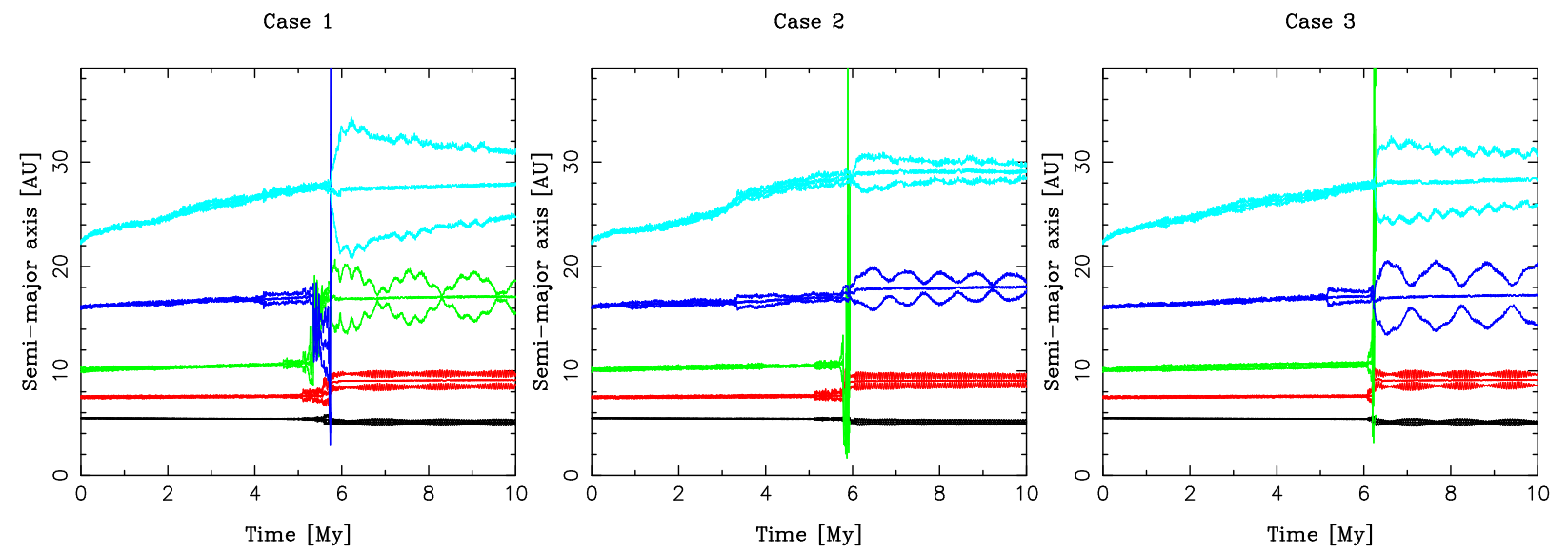
Fig. 3.- Initial conditions for Phase 1: Early Instability set (left) and Late Instability set (right). Black dots correspond to the MB population. Red dots correspond to the HG and TS populations (only in the Early Instability set). The big black dot represents Jupiter

EI set
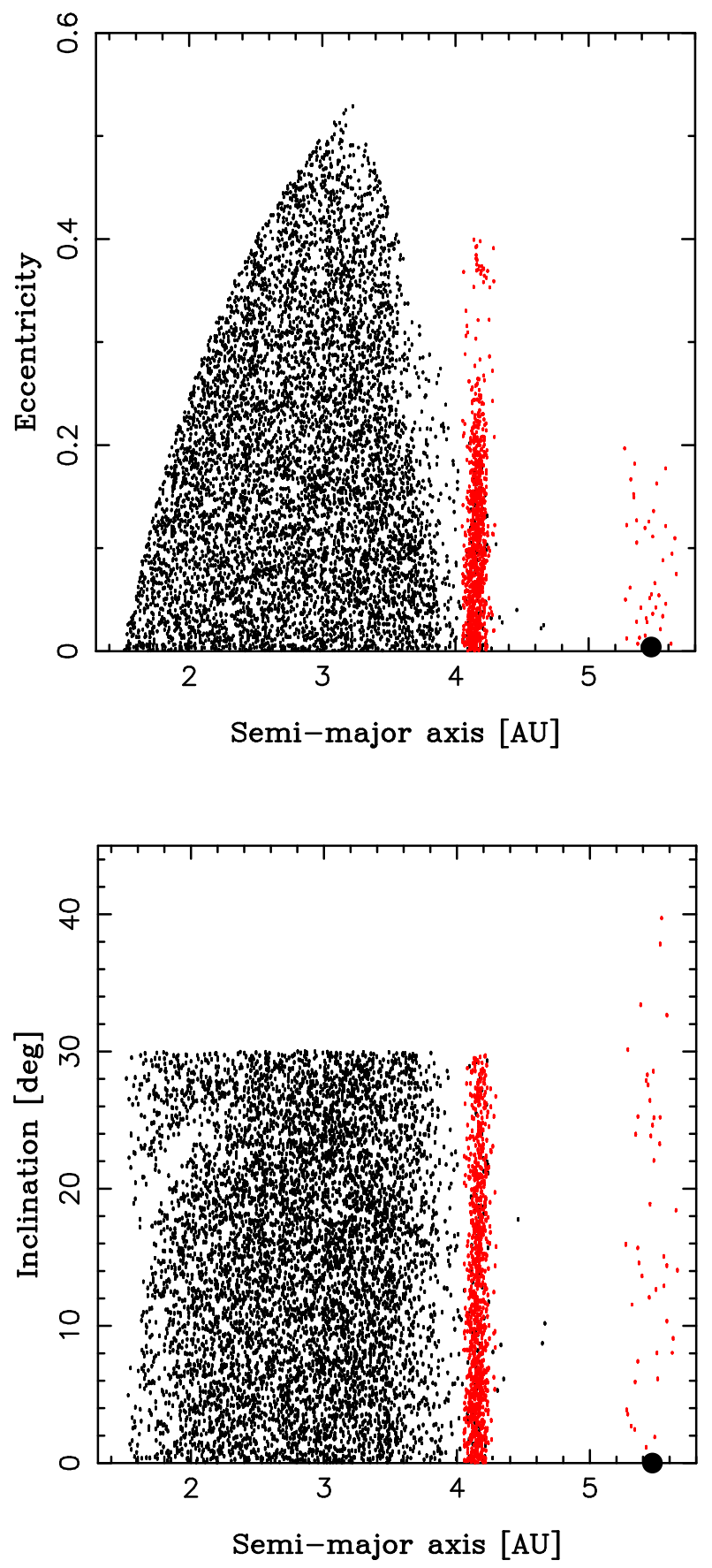

LI set
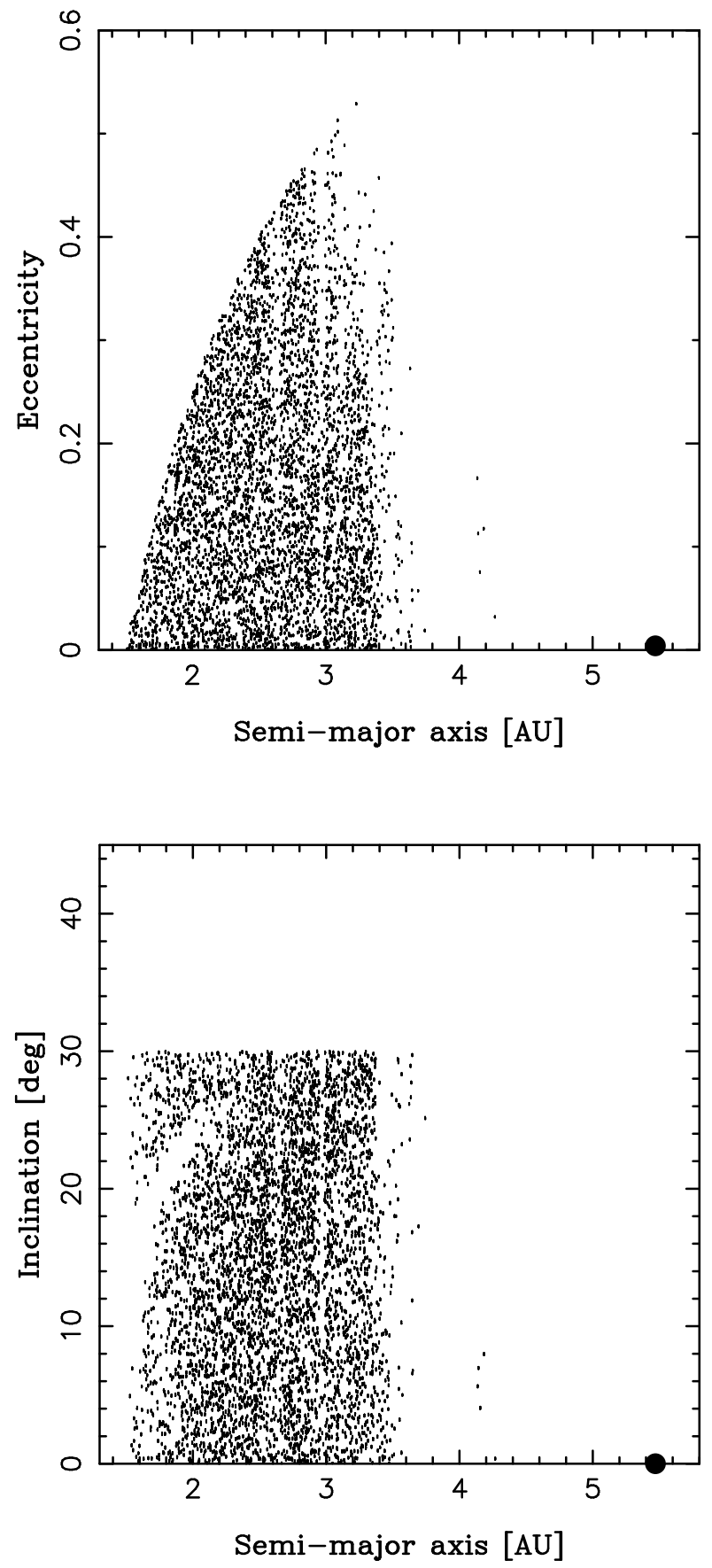
Fig. 4.- The distribution of the MB population in the Early Instability set, at the end of Phase 1 (migration Case 1). About $\sim 46000$ test particles (of the originally 102000 ) survived the instability. Their orbits are used as the initial conditions for Phase 2. The big dot represents Jupiter. The gap at $\sim 2$ au is due to the $\nu_{6}$ secular resonance.
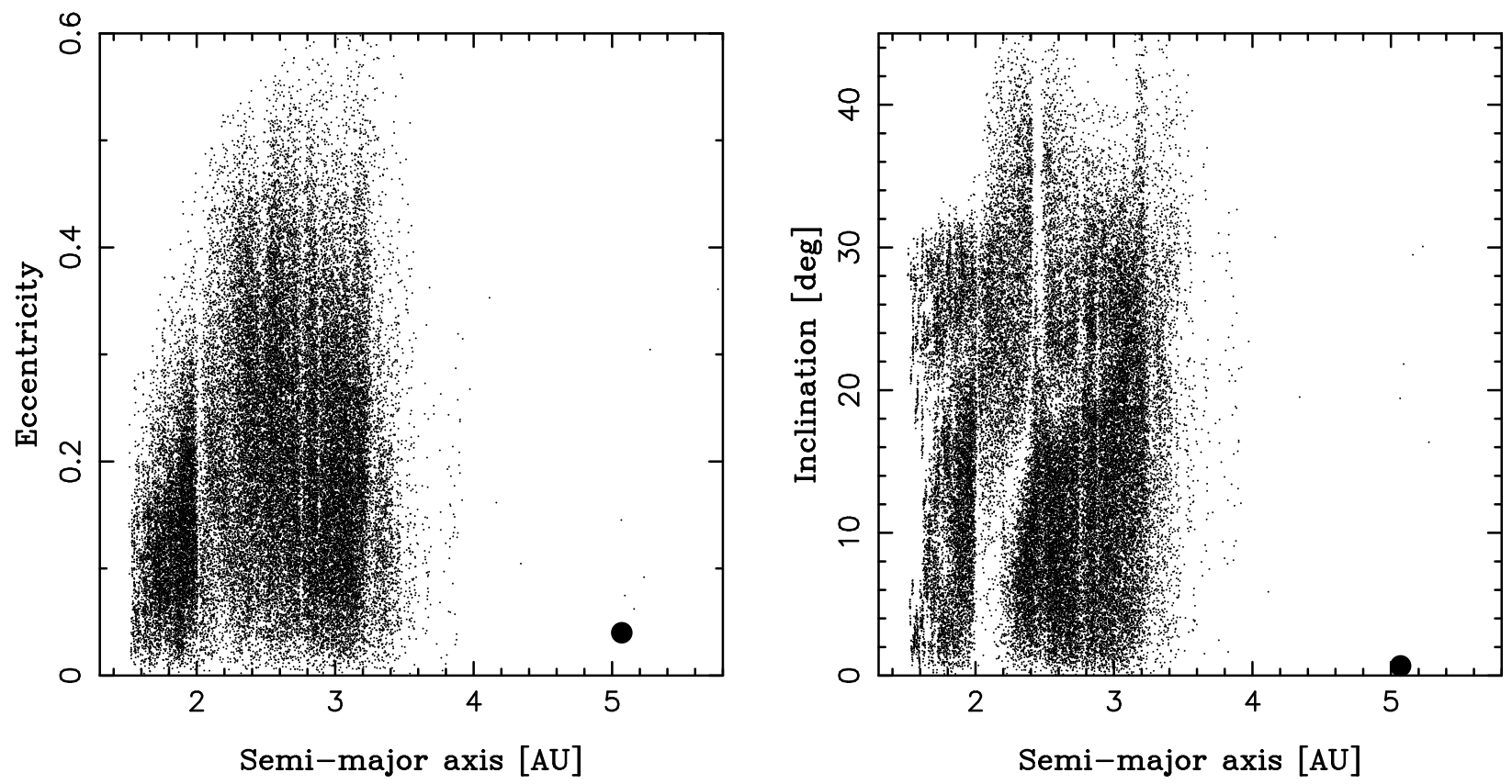
Fig. 5.- The tiny dots represent the distribution of the MB population in the Early Instability set, at the end of Phase 2 (migration Case 1). About $\sim 32000$ test particles (of the initially 46000 ) survived the residual migration. Test particles with $q<1.6$ au and $a<2.1$ au (tiny black dots) are not taken into account. Of the remaining $\sim 22000$ test particles (tiny cyan dots), we perform a random down-sampling to get the set of 1008 particles represented by the blue dots (note that particles with $a \geq 3.5$ au have not been down-sampled). These are used as initial conditions for Phase 3. In red, we indicate the location of the main mean motion resonances with Jupiter (left), and the location of the main secular resonances (right).
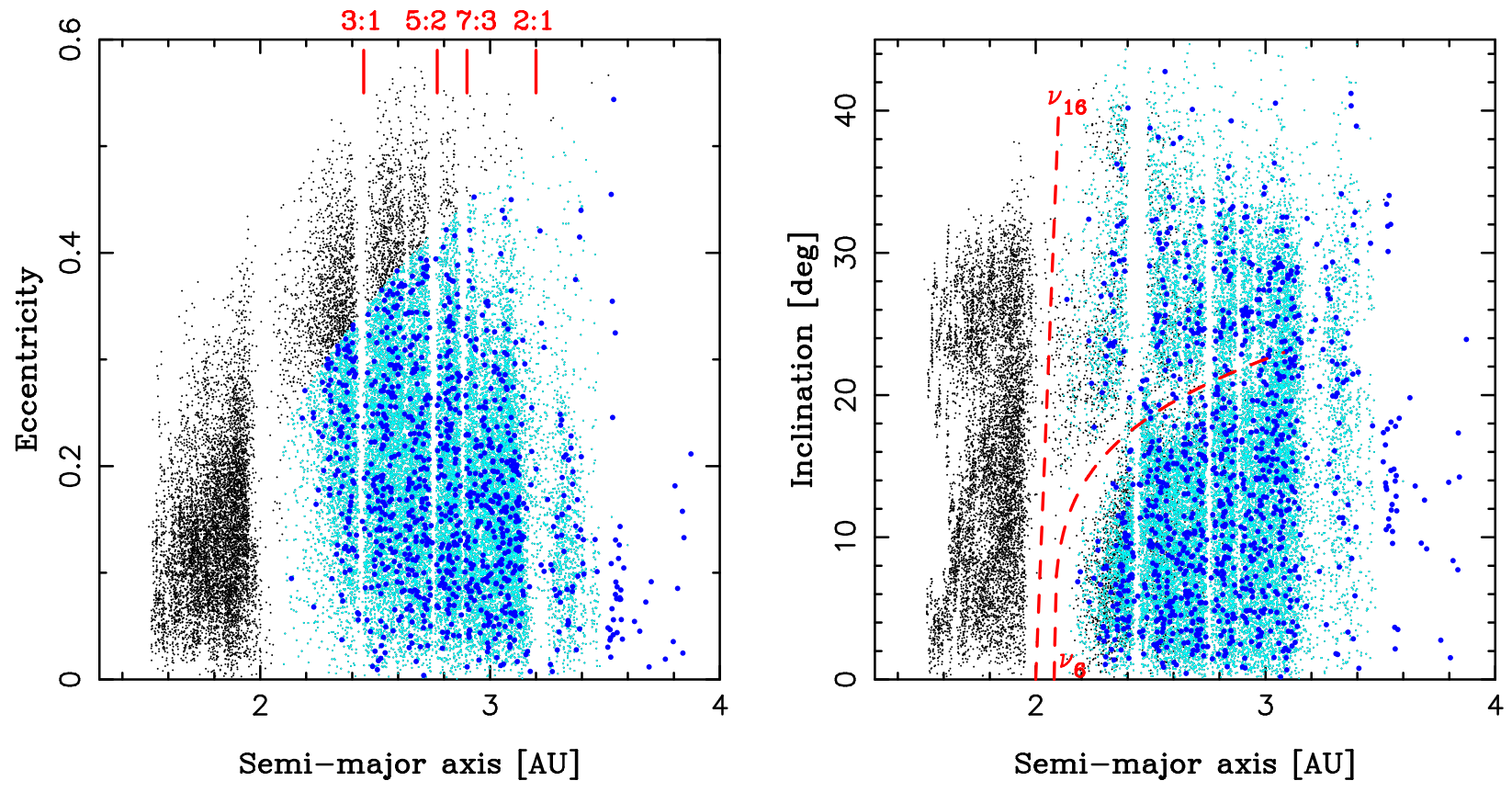
Fig. 6. - The blue dots represent the final distribution of 800 test particles in the MB population (Early Instability set, migration Case 1), at the end of Phase 3. The distribution of 574 real asteroids with $H \leq 9.7$ (red dots) is shown for comparison. The dotted-dashed line in panel (a) corresponds to $q=1.6 \mathrm{au}$. The dashed lines in panel (b) give the approximate location of the $\nu_{6}$ and $\nu_{16}$ secular resonances.
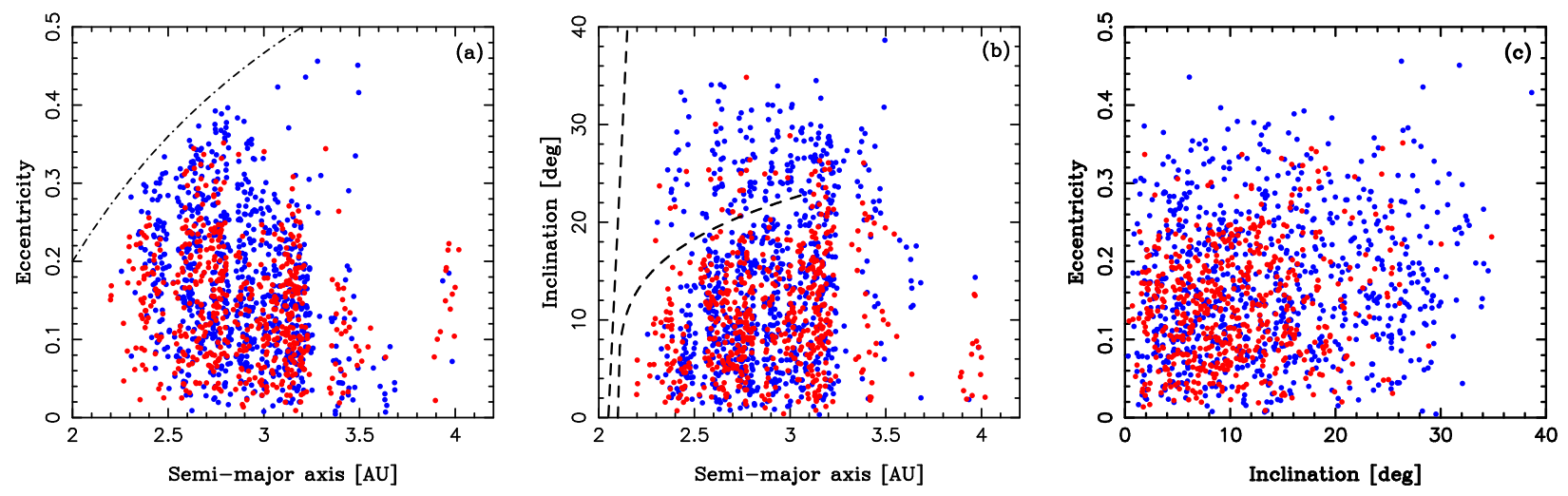

Fig. 7.- Cumulative distributions in $e$ and $I$, and histogram of the density distribution in a, comparing the real asteroids with $H \leq 9.7$ (full lines) to the simulated test particles at the end of Phase 3 (dashed lines and gray histogram). The simulation is the same presented in Fig. 6]
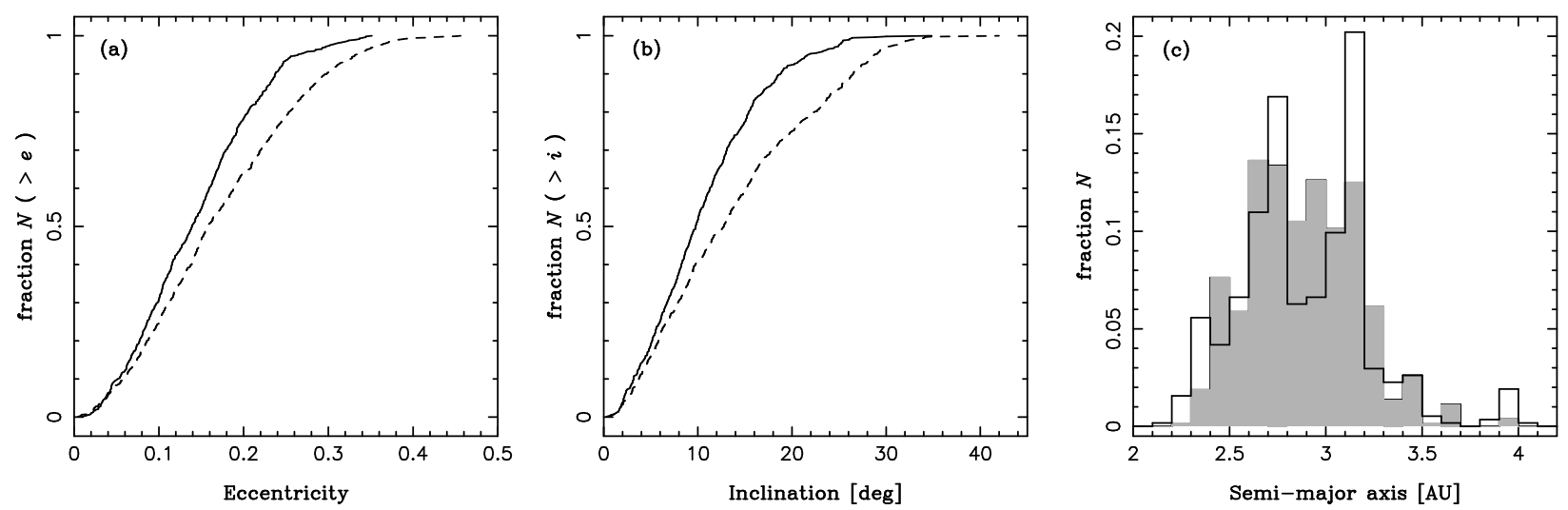
Fig. 8.- Comparison of the 2-D distribution in the $e, I$ plane between the test particles of the EI set at the end of Phase 3 and the current asteroid belt. The color scale gives the values of the two dimensional Kolmogorov-Smirnoff statistic $D_{\mathrm{KS}}$. Best fits are obtained for $D_{\mathrm{KS}} \leq 0.1$ (dark blue color). The white curve encloses the region where $D_{\mathrm{KS}}$ has the highest significance levels. The black pixels in the lower left corner of the plots correspond to values for which the K-S test could not be applied due to the small amount of data $(N<5)$. The abscissas give the parameter value of the assumed initial distribution in $I$ (i.e. at the beginning of Phase 1). The ordinates give parameter value of the assumed initial distribution in $e$. The top row corresponds to initial upper cutoff distributions. The bottom row corresponds to initial Rayleigh distributions. Each column corresponds to a different migration case.

Case 1
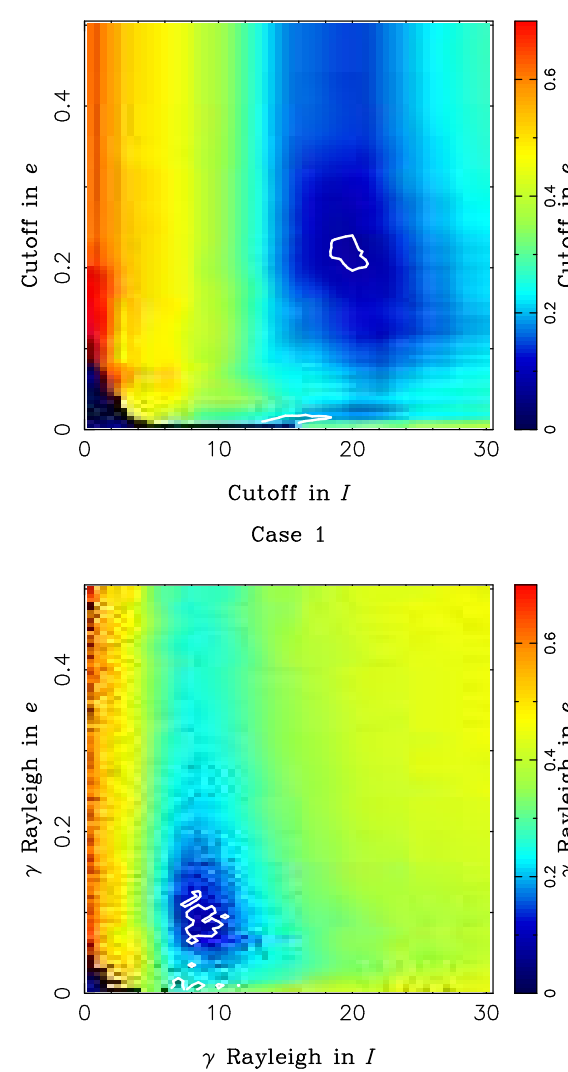

Case 2
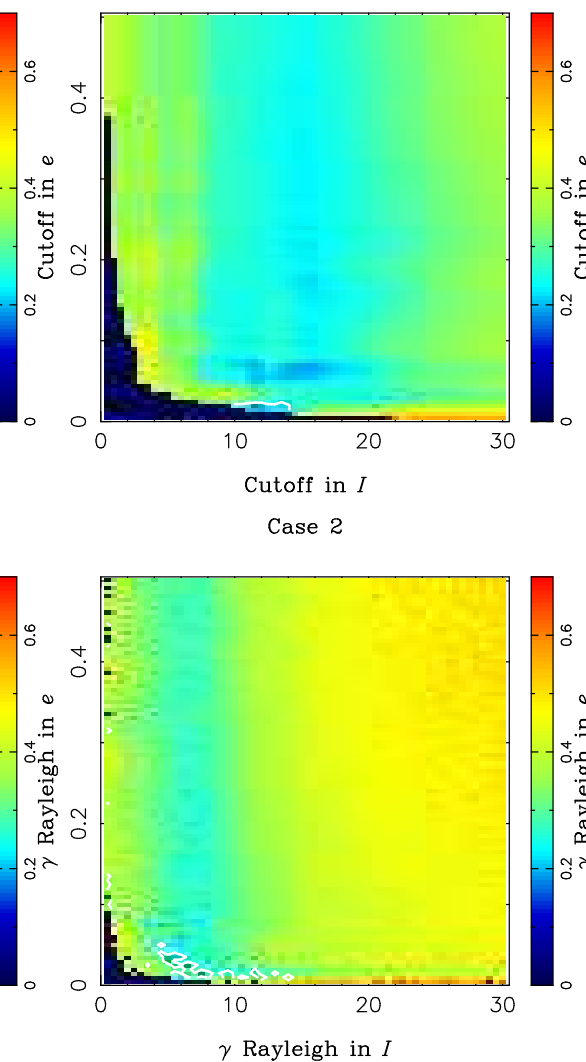

Case 3

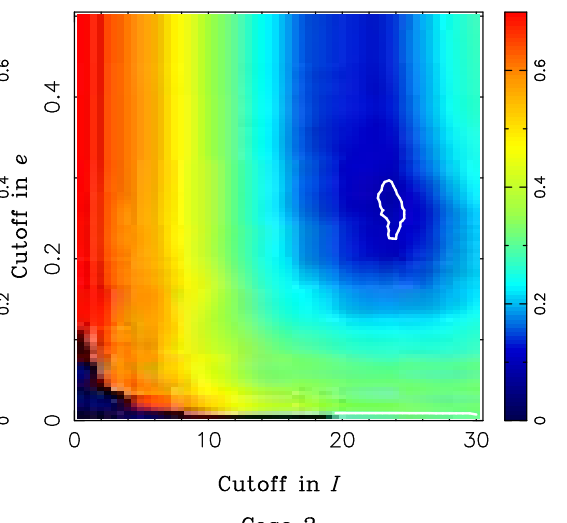

Case 3

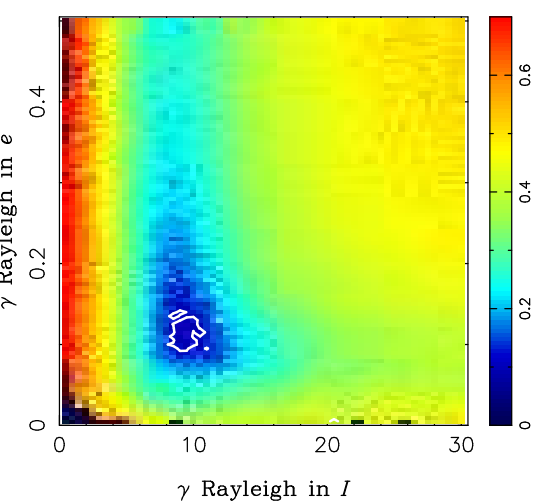


Fig. 9.- Similar as Fig. 8 but for the LI set. The top row corresponds to migration Case 1. The bottom row corresponds to migration Case 3. The left column shows initial upper cutoff distributions; middle column shows initial Rayleigh distributions, and right column shows initial Maxwell distributions.

Case 1

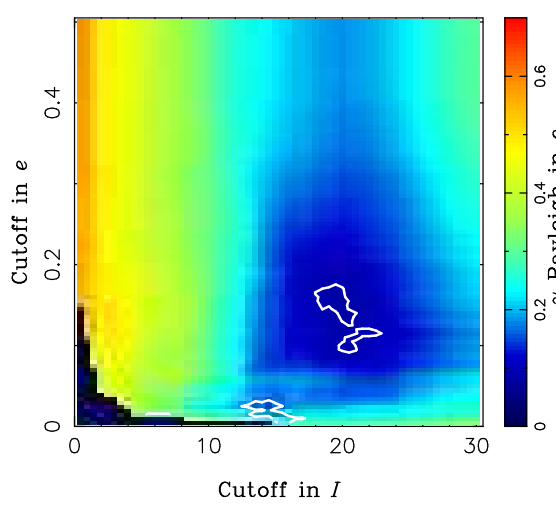

Case 3

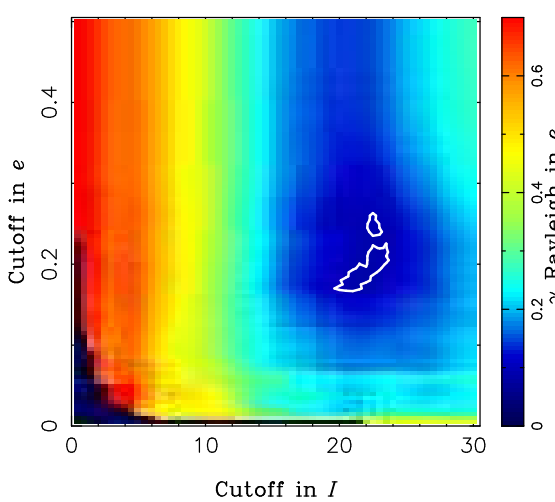

Case 1

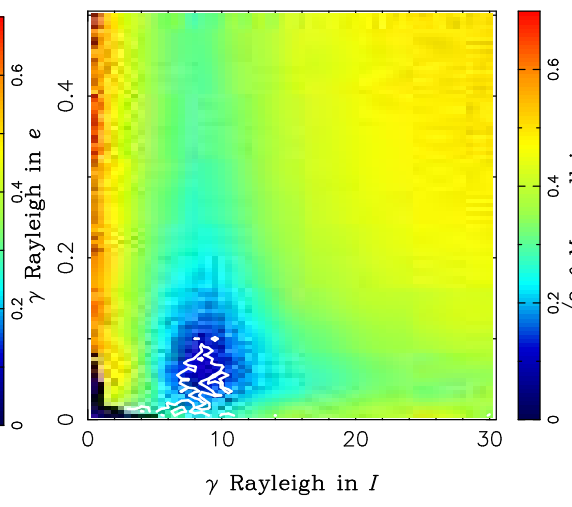

Case 3

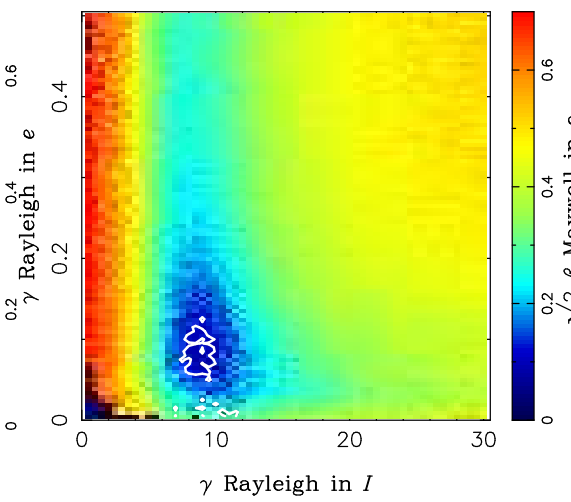

Case 1

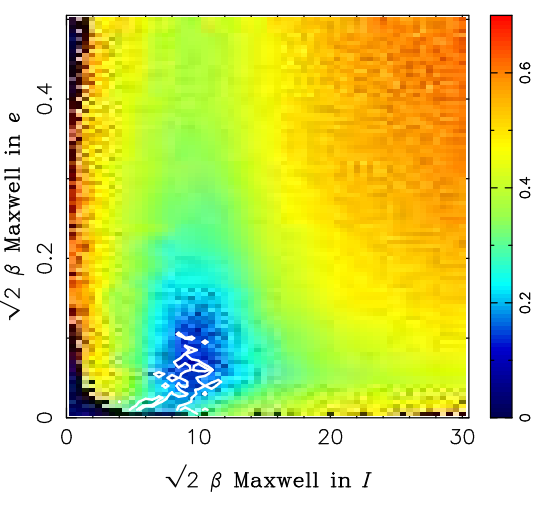

Case 3

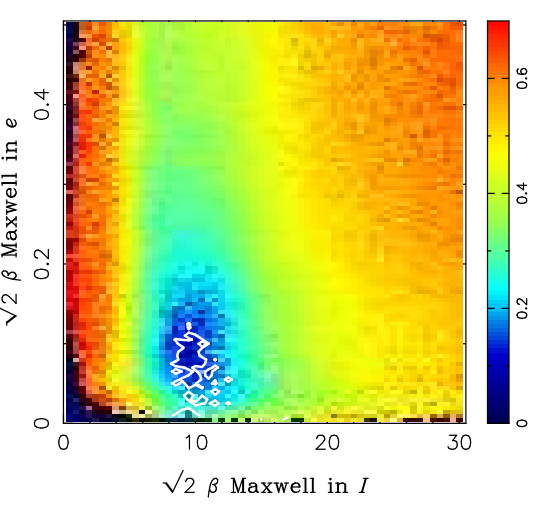


Fig. 10.- Similar to Fig. 8, but assuming initial distributions as those assumed for the Grand Tack. In the left column, the abscissas give the mode of an initial Rayleigh distribution in $I$, while the ordinates give the standard deviation of an initial Gaussian distribution in $e$, with fixed mean $(\mu=0.38)$. In the middle column, the abscissas give the mode of an initial Rayleigh distribution in $I$, while the ordinates give the mean of an initial Gaussian distribution in $e$, with fixed standard deviation $(\sigma=0.17)$. Finally, in the right column, the abscissas give the mean and the ordinates give the standard deviation of an initial Gaussian distribution in $e$, assuming an initial Rayleigh distribution in $I$ with fixed mode $\left(\gamma=10^{\circ}\right)$. The black triangle indicates the values reported by Walsh et al. (2012).

Case 1

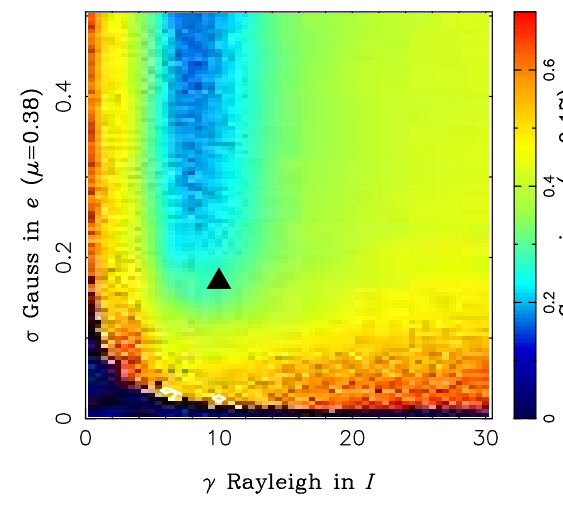

Case 3

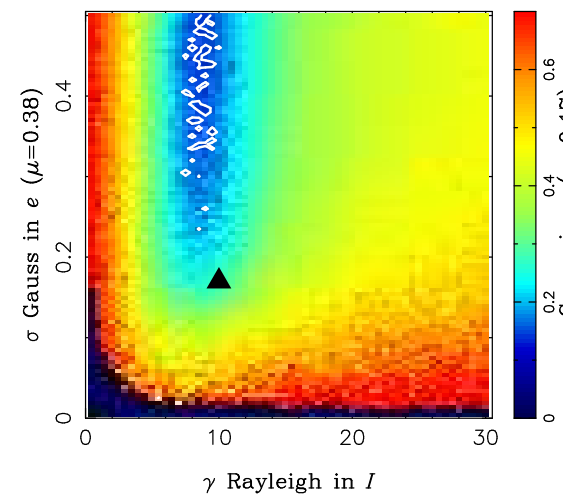

Case 1

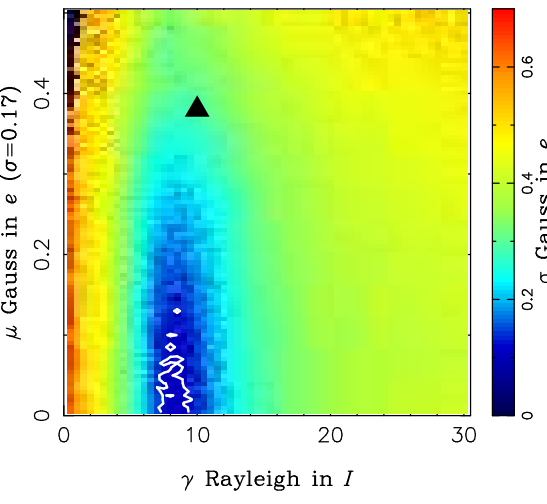

Case 3
Case 1

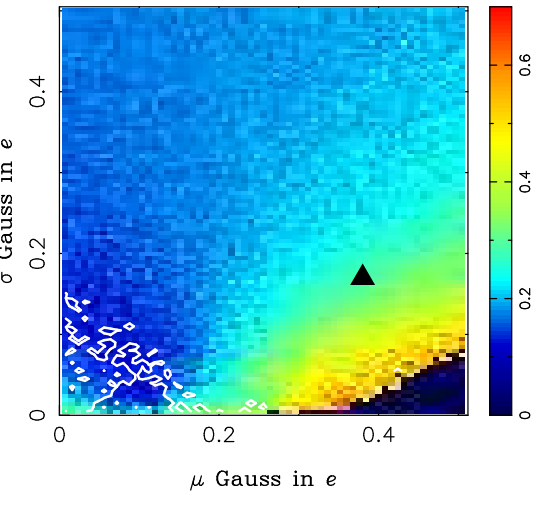

Case 3

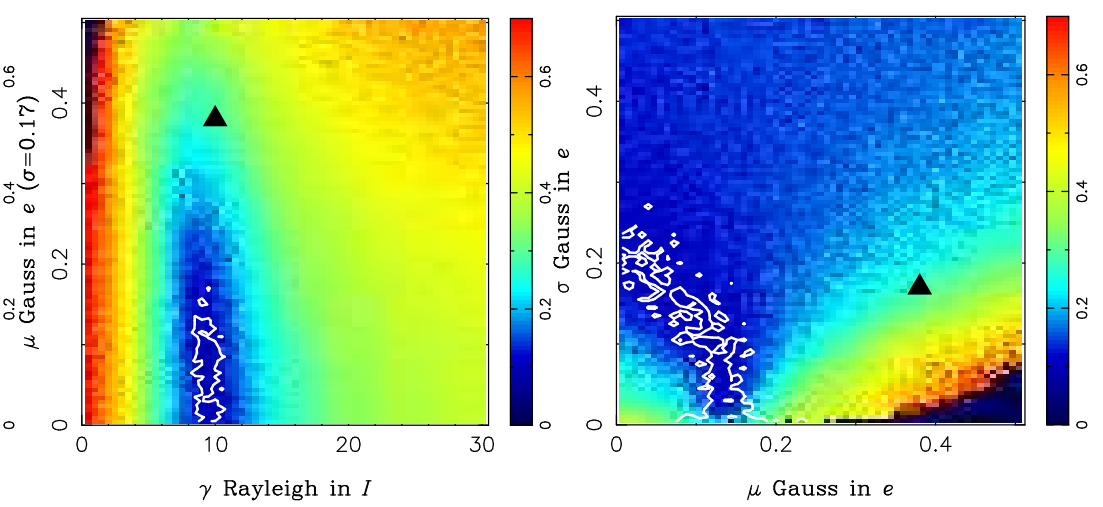


Fig. 11. - The same example shown in Fig. 7 (EI set, migration Case 1), but assuming initial distributions remapped into the nominal Grand Tack distributions $(\mu=0.38, \sigma=0.17$, and $\left.\gamma=10^{\circ}\right)$
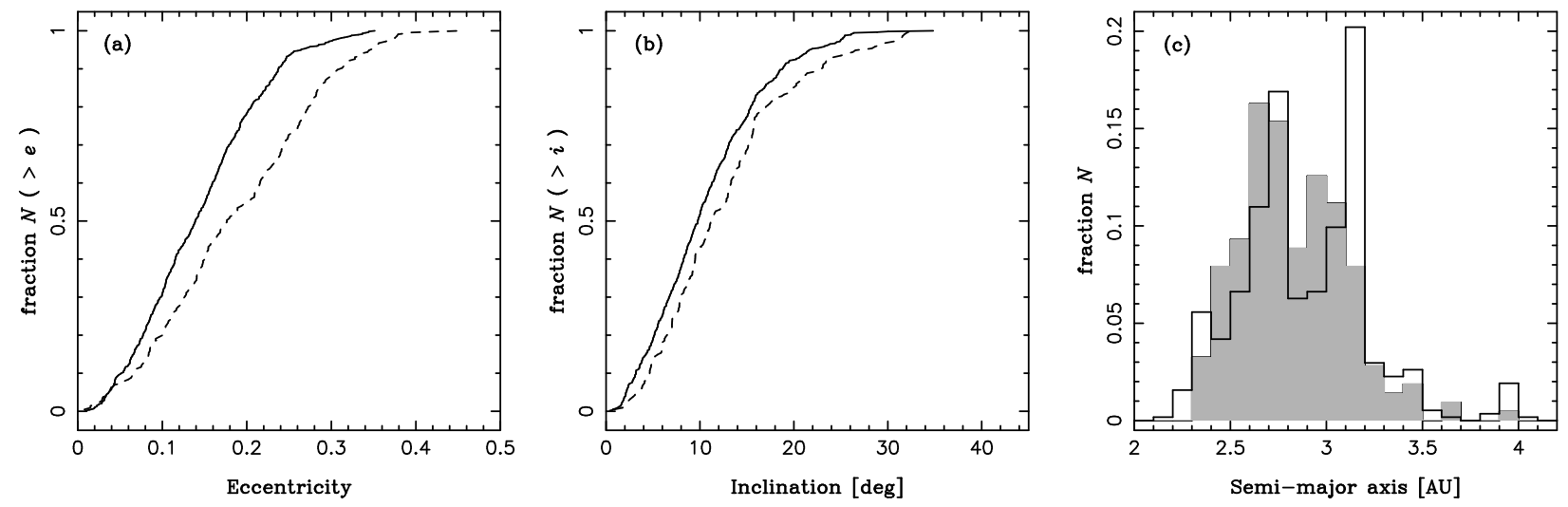
Fig. 12.- Results of the $\chi^{2}$ test (color scale) for the semi-major axis distribution of the EI set in the three migration cases considered here. The horizontal and vertical axes give the values of the parameters $a_{c}$ and $\varepsilon$ of the band cut initial distribution in $a$. A cut level $\varepsilon=0$ means that all the test particles within a band centered at $a_{c}$ have been removed. A cut level $\varepsilon=1$ means that no test particle has been removed. Good fits are obtained for $\chi^{2} \leq 0.14$ (note that $\chi^{2}$ is always larger than 0.1). Initially, $e$ and $I$ were assumed to follow the Rayleigh distributions with modes $\gamma=0.1$ and $10^{\circ}$, respectively. The black rectangle shown in migration Case 1 indicates a region where the fits are the best. Similar results were obtained for the LI set.
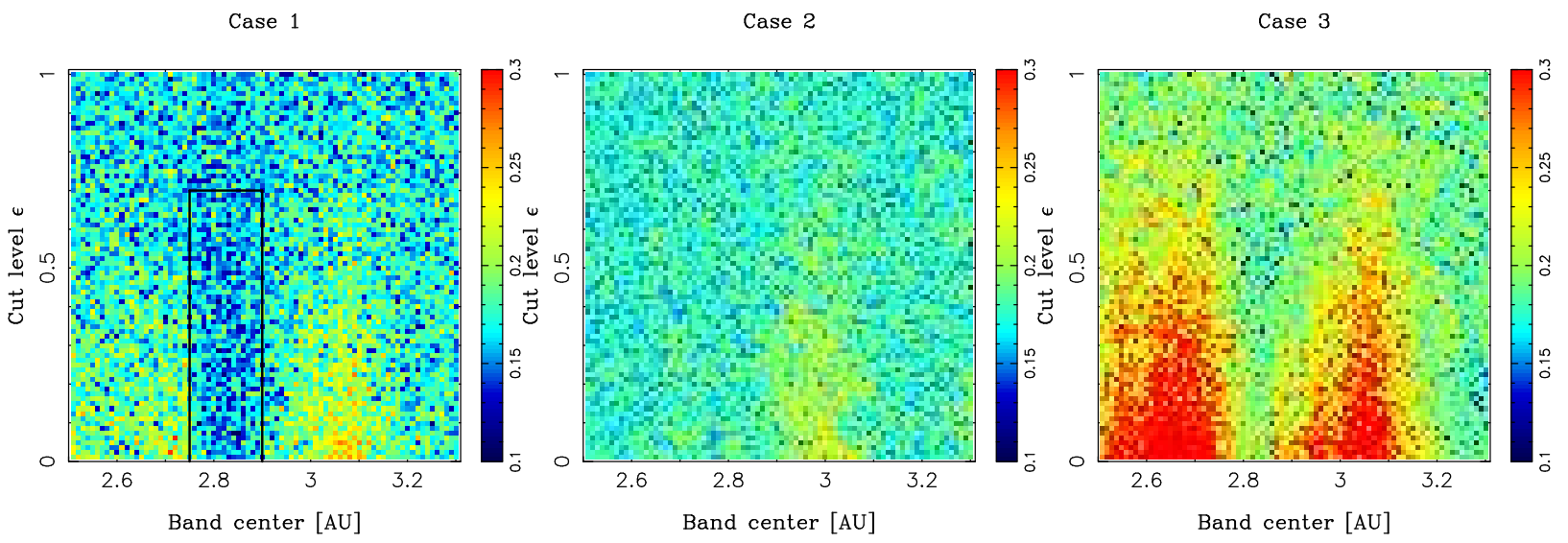
Fig. 13.- The same example shown in Fig. 7 (EI set, migration Case 1), but now assuming initial distributions remapped into the Rayleigh distributions in $e$ and $I$. The modes in $e$ and $I$ are $\gamma=0.1$ and $10^{\circ}$, respectively. No remapping of the distribution in $a$ has been applied. Note the larger depletion in the "pristine zone" (2.8 to $3.1 \mathrm{au}$ ) compared to Fig. 7.
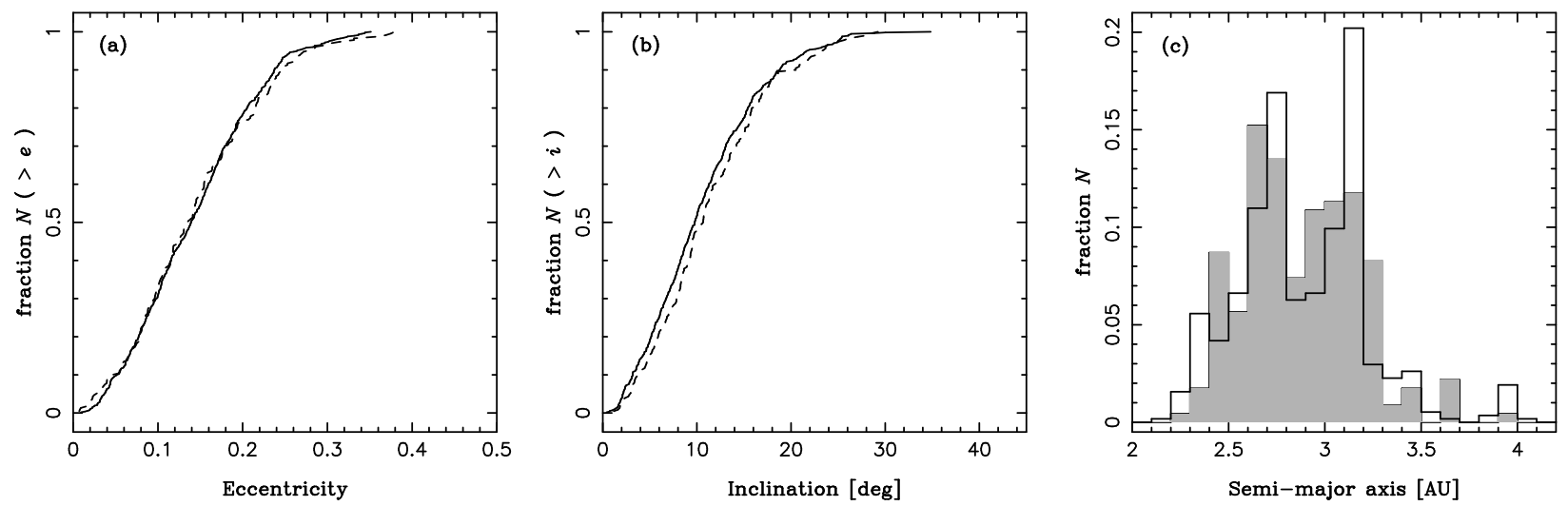
Fig. 14.- Top row: Distribution of the MB test particles that became implanted in the Hilda region (blue dots) at the end of Phase 1. Gray dots represent the MB population. Red dots are the implanted test particles that survived until the end of Phase 3. Bottom row: Initial distribution, at the beginning of Phase 1, of the MB test particles that become implanted in the Hilda region. Gray dots represent the whole MB initial population. This example corresponds to the EI set, migration Case 3.
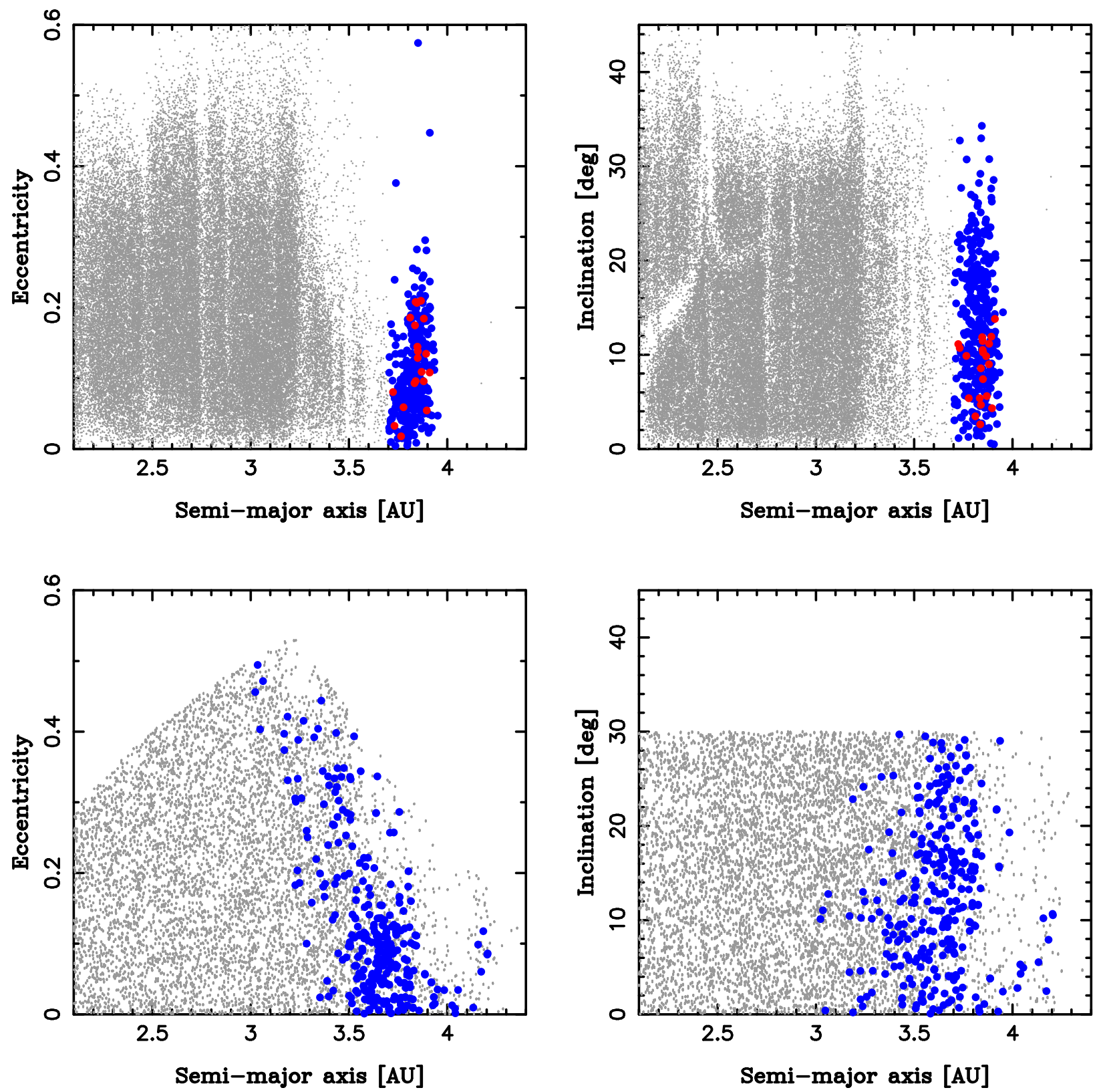
Table 1: Initial orbital elements of the five major planets.

\begin{tabular}{ccccc}
\hline \hline Planet & Mass $\left[M_{\mathrm{Jup}}\right]$ & $a[\mathrm{au}]$ & $e$ & $I\left[^{\circ}\right]$ \\
\hline Jupiter & 1.0 & 5.47 & 0.003 & 0.05 \\
Saturn & 0.299 & 7.45 & 0.011 & 0.02 \\
Ice \#1 & 0.053 & 10.11 & 0.017 & 0.11 \\
Ice \#2 & 0.053 & 16.08 & 0.006 & 0.07 \\
Ice \#3 & 0.053 & 22.17 & 0.002 & 0.05 \\
\hline \hline
\end{tabular}

Table 2: Statistic of surviving test particles during Phase 0.

\begin{tabular}{cccc}
\hline \hline$t[\mathrm{Myr}]$ & $\mathrm{MB}$ & $\mathrm{HG}$ & $\mathrm{TS}$ \\
\hline 10 & 6820 & 992 & 44 \\
500 & 4690 & 12 & 1 \\
\hline \hline
\end{tabular}

Table 3: The median, mean and maximum dispersion of orbital elements (absolute value of the difference between the elements at the beginning of Phase 1 and at the end of Phase 3), for each case of migration (EI set).

\begin{tabular}{|c|c|c|c|c|c|c|c|c|c|}
\hline & \multicolumn{3}{|c|}{$|\delta a|[\mathrm{au}]$} & \multicolumn{3}{|c|}{$|\delta e|$} & \multicolumn{3}{|c|}{$|\delta I|\left[^{\circ}\right]$} \\
\hline & Median & Mean & Max & Median & Mean & Max & Median & Mean & Max \\
\hline Case 1 & 0.064 & 0.114 & 0.707 & 0.069 & 0.112 & 0.370 & 2.16 & 4.25 & 22.38 \\
\hline Case 2 & 0.118 & 0.231 & 1.013 & 0.100 & 0.137 & 0.396 & 4.10 & 6.56 & 26.50 \\
\hline Case 3 & 0.022 & 0.072 & 0.466 & 0.057 & 0.095 & 0.398 & 1.71 & 3.16 & 19.37 \\
\hline
\end{tabular}

